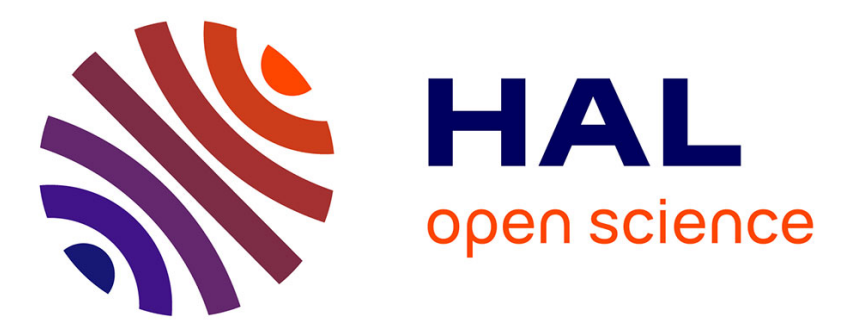

\title{
Complex conductivity in the presence of long range potential fluctuations. Application to the determination of the gap state density in undoped and boron doped a-Si : $\mathrm{H}$ films grown by CVD
}

Fernand-Michel Roche, B. Pistoulet, T. Soegandi

\section{To cite this version:}

Fernand-Michel Roche, B. Pistoulet, T. Soegandi. Complex conductivity in the presence of long range potential fluctuations. Application to the determination of the gap state density in undoped and boron doped a-Si: H films grown by CVD. Journal de Physique, 1988, 49 (11), pp.1933-1950. 10.1051/jphys:0198800490110193300 . jpa-00210873

\section{HAL Id: jpa-00210873 https://hal.science/jpa-00210873}

Submitted on 1 Jan 1988

HAL is a multi-disciplinary open access archive for the deposit and dissemination of scientific research documents, whether they are published or not. The documents may come from teaching and research institutions in France or abroad, or from public or private research centers.
L'archive ouverte pluridisciplinaire HAL, est destinée au dépôt et à la diffusion de documents scientifiques de niveau recherche, publiés ou non, émanant des établissements d'enseignement et de recherche français ou étrangers, des laboratoires publics ou privés. 


\title{
Complex conductivity in the presence of long range potential fluctuations. Application to the determination of the gap state density in undoped and boron doped a-Si : $\mathrm{H}$ films grown by CVD
}

\author{
F. M. Roche, B. Pistoulet and T. Soegandi(*) \\ Laboratoire d'Automatique et de Microélectronique de Montpellier associé au CNRS (UA 371), U.S.T.L., \\ Place Eugène bataillon, 34060 Montpellier Cedex, France
}

(Reçu le 7 avril 1987, révisé le 16 juin 1988, accepté le 29 juin 1988)

\begin{abstract}
Résumé. - Dans les semi-conducteurs compensés, à l'état cristallin ou à l'état amorphe, des fluctuations de potentiel s'étendant sur des distances grandes devant le libre parcours moyen des porteurs affectent de façon importante les propriétés de transport : elles agissent sur le déplacement des porteurs libres et changent le degré d'ionisation des centres profonds. L'amplitude maximale des fluctuations de potentiel varie avec le champ électrique appliqué, de sorte que la charge et la décharge de ces centres, à la fréquence du champ appliqué, contribuent à la conductivité complexe du matériau. Nous établissons l'expression de la conductivité complexe totale due au réseau, aux porteurs libres, et aux centres profonds, dans le cas général d'un semiconducteur compensé, c'est-à-dire présentant des fluctuations de potentiels. Ces expressions diffèrent considérablement de celles qui sont valables en l'absence de fluctuations. Il en résulte une nouvelle méthode de détermination de la densité d'états dans la bande interdite qui, contrairement aux techniques d'effet de champ, permet de séparer la contribution des états localisés de celle des porteurs des bandes. Cette méthode est appliquée à l'interprétation de nos mesures sur des films de a-Si : $\mathrm{H}$ déposés par CVD. Les valeurs calculées des parties réelle et imaginaire de la conductivité sont en accord avec les valeurs expérimentales dans de grands intervalles de température et de fréquence. Les variations de la conductivité complexe sont complètement expliquées par la seule influence des fluctuations de potentiel sur le déplacement des porteurs libres et sur l'ionisation des centres profonds. On ne met pas en évidence une conduction par sauts. La densité d'états présente une série de pics discrets éventuellement superposés à une distribution continue de plus faible densité.
\end{abstract}

\begin{abstract}
In compensated crystalline and amorphous semiconductors long range potential fluctuations (PF), at the scale of the mean free path, largely affect the transport properties : they act on free carriers and they change the average degree of ionization of deep centers. The maximum magnitude of PF varies with the applied field, and the corresponding charge and discharge of deep centers in the bulk, at the frequency of the applied field, contribute to the complex conductivity of the sample. The expressions of the total complex conductivity due to lattice, free carriers and deep centers, and of the complex differential capacitance of a MIS diode, are derived in the general case of a compensated semiconductor with potential fluctuations (PF). These expressions differ significantly from those commonly used when ignoring the existence of PF. This ends up in a new method for the determination of the distribution of gap states which, contrary to other methods, allows to separate the contribution of the localized states from the contribution of band carriers. This method is applied to the interpretation of our data on a-Si: $\mathrm{H}$ films grown by CVD. The real and the imaginary parts of the conductivity can be fitted in wide ranges of temperatures and frequencies. The variations of the complex conductivity are completely explained by the action of PF on the transport of free carriers and on the ionization of deep centers. There is no evidence of hopping transport. The density of states shows a discrete series of peaks possibly superposed to a lower continuous density.
\end{abstract}

(*) Present address : LEN-LIPI, Jalan Soekarno Hatta 480/205 A 10254 Bandung, Indonesia. 


\section{Introduction.}

Since the classical theory of Debye [1], developed for polar liquids, the study of dispersion and absorption in dielectrics, and more recently in semi-insulators, and in compensated crystalline and amorphous semiconductors, has been the subject of a large amount of experimental and theoretical work. In order to fit the permittivity and conductivity data versus frequency and temperature, empirical expressions have been proposed by Cole and Cole [2], Davidson and Cole [3], Jonscher [4], Macdonald [5] and others. However, in the absence of a precise microscopic representation, these models do not generally allow one to obtain worthwhile information on the polarization and conduction processes and on the density of states. In the case of a-Si : $\mathrm{H}$ various methods of determination of the gap state density have been used : junction capacitance, field effect (FE), space charge limited currents (SCLC), thermostimulated currents, sweep-out technique [6, 7], electron spin resonance (ESR), and others. It is observed [8] that a rough agreement is found near the gap center, but large discrepancies still exist below and above.

In nearly all their papers, the authors, in order to explain the data, assume implicitly, and without any attempt of justification, that the material is free from long range potential fluctuations (PF), and can thus be considered as microscopically homogeneous at the scale of the band carrier mean free path. However the non-uniform distribution of ionized shallow centers in the grown solid creates long range potential fluctuations, which are nearly unscreened in highly compensated crystalline and amorphous semiconductors, due to the low carrier density. The existence of PF was first considered by Wannier [9] in insulators, by Shockley and Bardeen [10] in crystalline semiconductors, by Fritzsche [11] in amorphous semiconductors, and by Shklovskii and Efros $[12,13]$ who have shown that randomly distributed donors and acceptors create large scale PF of magnitude comparable with the forbidden bandwidth. Pistoulet et al. [14-16] derived general expressions of the dc transport coefficients by band carriers in the presence of PF; dc conductivity activation energy and preexponential factor, photoconductivity, thermopower are drastically changed by PF. The expression of the complex conductivity $\sigma_{\mathrm{ac}}(\omega, T)+i \omega \varepsilon_{\mathrm{ac}}(\omega, T)$ from dc to very high frequencies, when the effect of PF on band carriers is considered, was derived by Pistoulet, Roche, Abdalla [17] (PRA) ; the validity of the model was numerically confirmed by Soegandi, Roche, Pistoulet [18]. It was shown [17] that the data of Pollak and Geballe [19], Abkowitz et al. [20], Long and Balkan [21] do not demonstrate hopping conduction but result simply from the effect of PF on band carriers.
Data of Moustakas and Weiser [22], Pfister [23] were also simply explained [24].

However, most of the authors studying amorphous semiconductors still focus their attention exclusively on short range fluctuations which, as shown by Anderson [25], Mott [26], Cohen-Fritzsche-Ovshinsky [27], produce significant changes in the density of states, while long range PF leave the local density of states unchanged [28]. But, in the presence of PF, the Fermi level is crossing the energy levels of various species of centers in a large number of places in the sample, so that the degree of ionization of the centers depends on the magnitude of PF. Moreover when the sample is submitted to an a.c. field, the field dependence of PF magnitude [29] is responsible for the charge and discharge of deep centers which contribute significantly to bulk conductivity and permittivity, and to surface capacitance. This fact has serious consequences for the determination of the gap state density.

Since the first field effect experiments of Spear et al. [30, 31], a large number of methods have been proposed for the determination of the density of states in amorphous semiconductors. Yet very few methods appear to be able to distinguish clearly if the experimental data correspond to: i) a material free from PF with band tails and wide peaks in the gap state density or ii) a material with long range $\mathrm{PF}$, having in each domain sharp band edges, and discrete levels or narrow peaks in the gap. It has been shown [24] that the average charge density in a plane parallel to the surface of the sample is similar in both cases. Therefore, in field effect and in capacitance measurements, discrete levels in the presence of PF have the same effect as wide peaks in the absence of PF, as sketched in figure 2 of reference [14], so the effect of free carriers located in deep wells cannot be neglected. Similarly, transient capacitance spectroscopy and transient voltage spectroscopy only give access to average quantities ; as the local distance from the surface of the cross over point varies with the local potential, similar data are obtained by these techniques in cases i) and ii). Optical methods seem to be more suitable for the determination of the local properties of the material. In subgap optical absorption experiments however, the absorption depends on the energy of deep states relative to the Fermi level so, when averaging over the sample, the effect of PF on discrete levels is not easily distinguished from that of a continuous density of states. Luminescence is also sensitive to the presence of PF, as shown by Rehm et al. [32] who reported luminescence red shifts in superlattices due to tunnel assisted recombination. Photoconductivity [15b], space charge limited currents, and thermostimulated currents (which involve transport processes), are also most dependent on the presence of PF. It is therefore clear that, whatever the technique 
used, it is necessary to take into account the effect of PF when calculating the density of states from the data.

In the present paper a general theory of conductivity, permittivity and surface capacitance in the presence of PF is developed. It is shown in section 2 that these quantities can be precisely related to the physical characteristics of the material, i.e. the densities and binding energies of the centers, the magnitude of PF and their field dependence. No special assumption is made on the local band structure of the material, characterized by band edges $E_{\mathrm{c}}, E_{\mathrm{v}}$ and $a$ priori arbitrary and unknown distribution of deep centers and gap states. The PRA theory [17] is completed and generalized by taking into account the effect of wells and hills of maximum magnitude. In section 3 , our measurements on undoped and boron doped CVD a-Si : $\mathrm{H}$ films versus temperature and frequency are reported. By fitting the data, it is shown that the gap state density consists of a discrete series of peaks, possibly broadened, and that hopping conduction, if present, is completely hidden by the other processes.

In the following only extrinsic semiconductors with unipolar conductivity are considered. Except specific mention, the notation is that of reference [17].

2. Theory of bulk ac complex conductivity, and of surface capacitance, in the presence of PF.

2.1 MODEL. - The compensated (crystalline or amorphous) semiconductors considered in this paper are assumed to be macroscopically homogeneous and isotropic, i.e. filamentary structures, heterostructures, and superlattices are excluded. According to this assumption, transport coefficients measured on any workable sample, even of very small size, do not depend on its location nor on its orientation. We focus our attention on extrinsic semiconductors, in which only one band is appreciably populated, so that the fluctuations acting on band carriers are those of the band edge of the populated band. Thus covariant fluctuations of the band edges resulting from the non-uniform distribution of ionized impurities, or contravariant fluctuations resulting from band gap variations due to local departure from stoechiometry or other causes, have similar effects on the carriers of the populated band. The main difference between covariant and contravariant variations of the band edges concerns the ionization probability of deep centers. Often however, the semiconductors considered are far from intrinsic, so the Fermi level is close to one band and the contravariant part of the band edge fluctuations does not significantly change the ionization probability of the levels. Therefore, although only PF leaving the gap $E_{\mathrm{G}}$ unchanged are considered in the following, the validity of the model is not strictly limited to this case.

Long range $\mathrm{PF}$ are defined as fluctuations of spatial extent larger than the band carrier mean free path, but much smaller than the dimensions of the sample. Any measurable sample is thus constituted $[14,15,17]$ by a $3 \mathrm{D}$ mosaic of a large number of elementary domains, each domain being characterized by a narrow range $\left(E_{\mathrm{c}}, E_{\mathrm{c}}+\mathrm{d} E_{\mathrm{c}}\right)$ of the conduction band edge energy. In transport experiments, the currents and the voltages are not measured locally in each domain, but through and across the whole sample. Therefore, the only measurable quantities are averaged values over the entire assembly of domains of a sample. If $X\left(E_{\mathrm{c}}\right)$ is the local value of a physical transport variable, the quantity $X$ experimentally measurable in a real sample can be expressed as :

$$
X=\int_{E_{\mathrm{c} \min }}^{E_{\mathrm{c} \max }} X\left(E_{\mathrm{c}}\right) P\left(E_{\mathrm{c}}\right) \mathrm{d} E_{\mathrm{c}}
$$

where $P\left(E_{\mathrm{c}}\right)$ is the probability of finding the band edge at energy $E_{\mathrm{c}}$. As in previous papers [14, 15, 17], it is assumed that the non-uniform distribution of ionized centers (mainly shallow impurities) produces a Gaussian distribution of PF, of standard deviation $\Gamma / \sqrt{2}$, which has its maximum at some value $E_{\mathrm{c} 0}$ of $E_{\mathrm{c}}$ :

$$
P_{\mathrm{G}}\left(E_{\mathrm{c}}\right)=\frac{1}{\Gamma \sqrt{\pi}} \exp \left(-\left(\frac{E_{\mathrm{c}}-E_{\mathrm{c} 0}}{\Gamma}\right)^{2}\right) .
$$

In fact, this Gaussian distribution is truncated [16, 17] at energies $E_{\mathrm{c} 0}-g_{\mathrm{c}} \Gamma$ and $E_{\mathrm{c} 0}+g_{\mathrm{v}} \Gamma$, where $g_{\mathrm{c}}, g_{\mathrm{v}}$ are positive numbers generally smaller than 3 , because the few electrons existing in the conduction band of the compensated semiconductor accumulate in the deepest potential wells, and the few holes in the highest hills, thus producing a local partial screening of the largest PF. Figure 1 represents the surface $E_{\mathrm{c}}(x, y)$ showing the spatial variations of the conduction band edge in a plane $x \mathrm{O} y$. Wells and hills of maximum magnitude $g_{\mathrm{c}} \Gamma$ and $g_{\mathrm{v}} \Gamma$ respectively play a significant role, particularly in the local ionization of deep centers. Their probabilities $P_{\delta}$ correspond to the truncated parts of the Gaussian and are represented by two $\delta$ functions centered at $E_{\mathrm{c} 0}-g_{\mathrm{c}} \Gamma$ and $E_{\mathrm{c} 0}+g_{\mathrm{v}} \Gamma$, and equal to :

$$
\begin{aligned}
& P_{\delta}\left(E_{\mathrm{c} 0}-g_{\mathrm{c}} \Gamma\right)=\frac{1}{\sqrt{\Pi}} \int_{g_{\mathrm{c}}}^{\infty} \exp \left(-u^{2}\right) \mathrm{d} u ; \\
& P_{\delta}\left(E_{\mathrm{c} 0}+g_{\mathrm{v}} \Gamma\right)=\frac{1}{\sqrt{\Pi}} \int_{g_{\mathrm{v}}}^{\infty} \exp \left(-u^{2}\right) \mathrm{d} u .
\end{aligned}
$$

The total probability function consists of the truncated Gaussian function $P_{\mathrm{G}}$ and of the two functions 


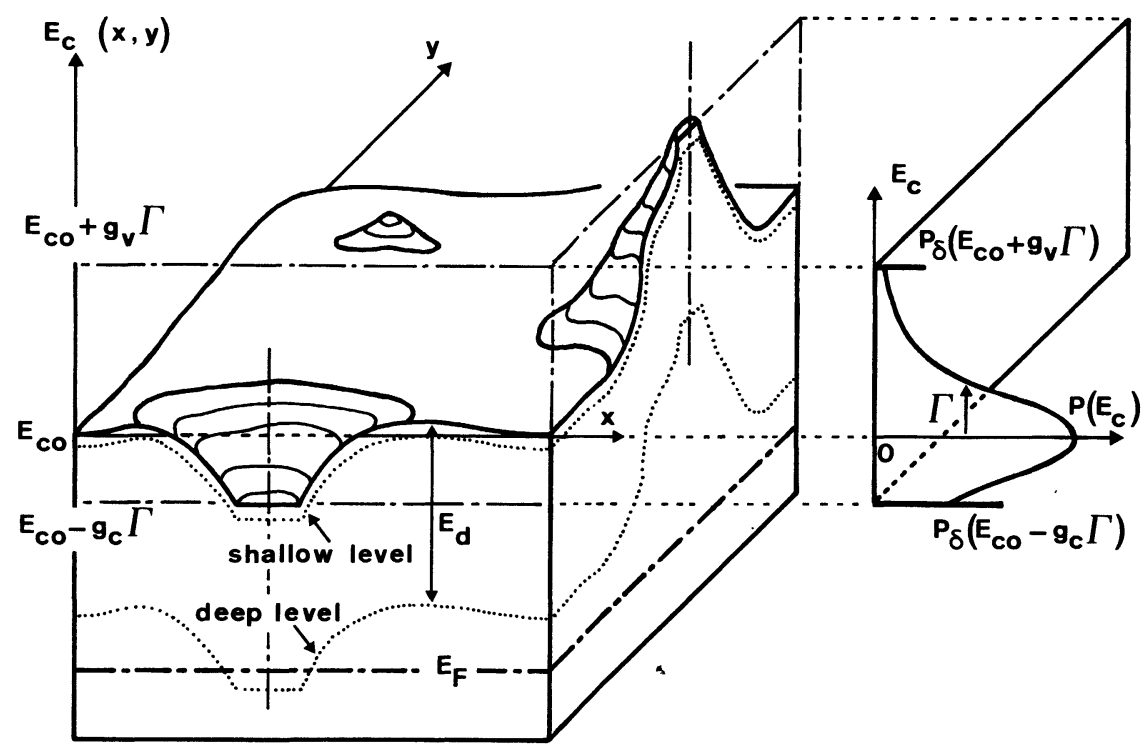

Fig. 1. - Conduction band edge $E_{\mathrm{c}}(x, y)$ in the presence of $\mathrm{PF}$, and probability $P\left(E_{\mathrm{c}}\right)$.

$P_{\delta}$. As the maximum depth of wells is proportional to temperature at low temperatures [17], the relative importance of the quantities $P_{\delta}$ increases as $T$ decreases. Considering [17] that the total probability $\alpha$ of wells can possibly differ from the total probability $(1-\alpha)$ of hills with $0<\alpha<1$, the total distribution function is equal to $\alpha P\left(E_{\mathrm{c}}\right)$ when $E_{\mathrm{c}}<E_{\mathrm{c} 0}$ and to $(1-\alpha) P\left(E_{\mathrm{c}}\right)$ when $E_{\mathrm{c}}>E_{\mathrm{c} 0}$. According to equations (1 to 3 ), the average value $X$ of a transport variable $X\left(E_{\mathrm{c}}\right)$ is now expressed as :

$$
\begin{aligned}
& X=\frac{2}{\sqrt{\Pi}}\left(\alpha \int_{-g_{\mathrm{c}}}^{0} X(u) \exp \left(-u^{2}\right) \mathrm{d} u+(1-\alpha) \int_{0}^{g_{\mathrm{v}}} X(u) \exp \left(-u^{2}\right) \mathrm{d} u+\right. \\
& \left.\quad+\alpha X\left(-g_{\mathrm{c}}\right) \int_{g_{\mathrm{c}}}^{\infty} \exp \left(-u^{2}\right) \mathrm{d} u+(1-\alpha) X\left(g_{\mathrm{v}}\right) \int_{g_{\mathrm{v}}}^{\infty} \exp \left(-u^{2}\right) \mathrm{d} u\right)
\end{aligned}
$$

where

$$
u=\frac{E_{\mathrm{c}}-E_{\mathrm{c} 0}}{\Gamma}
$$

In the presence of PF local neutrality does not exist, but in macroscopically homogeneous semiconductors the average densities of carriers and of ionized centers (species $i, j$ ) are related by the average neutrality equation $[16,17]$ :

$$
n-p=\sum_{i} N_{\mathrm{d} i}^{+}-\sum_{j} N_{\mathrm{aj}}^{-}:
$$

In this equation, the total average band carrier densities $n$ and $p$ are calculated according to equation (4). If we write

$$
n_{00}=N_{\mathrm{c}} \exp \left(\frac{E_{\mathrm{F}}-E_{\mathrm{c} 0}}{k T}\right)=n_{i}^{2} / p_{00}
$$

the local densities $n(u)$ and $p(u)$ to be introduced in equation (4) are :

$$
\begin{aligned}
& n(u)=n_{00} \exp \left(-\frac{u \Gamma}{k T}\right) ; \\
& p(u)=p_{00} \exp \left(\frac{u \Gamma}{k T}\right) .
\end{aligned}
$$

The local probabilities of ionization of donor $(i)$ and acceptor $(j)$ levels, at respective energies $E_{\mathrm{d} i}$, $E_{\mathrm{aj}}$ below $E_{\mathrm{c}}$ are classically written as :

$$
\begin{aligned}
& N_{\mathrm{d} i}^{+}(u) / N_{\mathrm{d} i}(u)=\left(1+g_{\mathrm{d} i} \exp \left(\frac{E_{\mathrm{F}}-E_{\mathrm{c}}(u)+E_{\mathrm{d} i}}{k T}\right)\right)^{-1}=\left(1+M_{i}(-u)\right)^{-1} \\
& N_{\mathrm{a} j}^{-}(u) / N_{\mathrm{a} j}(u)=\left(1+\frac{1}{g_{\mathrm{a} j}} \exp \left(\frac{E_{\mathrm{c}}(u)-E_{\mathrm{a} j}-E_{\mathrm{F}}}{k T}\right)\right)^{-1}=\left(1+\frac{1}{M_{j}(-u)}\right)^{-1}
\end{aligned}
$$


with :

$$
M_{i}(-u)=g_{\mathrm{d} i} \frac{n_{00}}{N_{\mathrm{c}}} \exp \left(\frac{E_{\mathrm{d} i}-u \Gamma}{k T}\right) ; \quad M_{j}(-u)=g_{\mathrm{a} j} \frac{n_{00}}{N_{\mathrm{c}}} \exp \left(\frac{E_{\mathrm{aj}}-u \Gamma}{k T}\right)
$$

The average density $N_{\mathrm{d} i}^{+}$of ionized donors appearing in equation (6) is obtained from equations (4) and (9). We assume that the density $N_{\mathrm{d} i}$ of deep centers is uniform, or at least not appreciably correlated to the distribution of shallow centers creating the PF, so that $N_{\mathrm{d} i}$ is independent of $u$, and the average density $N_{\mathrm{d} i}^{+}$can be written :

$$
\begin{aligned}
N_{\mathrm{d} i}^{+}=\frac{2 N_{\mathrm{d} i}}{\sqrt{\pi}}\left\{\alpha\left(\int_{0}^{g_{\mathrm{c}}} \frac{\exp \left(-u^{2}\right)}{1+M_{i}(u)} \mathrm{d} u+\frac{\int_{g_{\mathrm{c}}}^{\infty} \exp \left(-u^{2}\right) \mathrm{d} u}{1+M_{i}\left(g_{\mathrm{c}}\right)}\right)+\right. \\
\left.+(1-\alpha)\left(\int_{0}^{g_{\mathrm{v}}} \frac{\exp \left(-u^{2}\right)}{1+M_{i}(-u)} \mathrm{d} u+\frac{\int_{g_{\mathrm{v}}}^{\infty} \exp \left(-u^{2}\right) \mathrm{d} u}{1+M_{i}\left(-g_{\mathrm{v}}\right)}\right)\right\} .
\end{aligned}
$$

A similar expression is easily derived for $N_{\mathrm{aj}}^{-} / N_{\mathrm{aj}}$. These relations generalize equations $(19,20)$ of reference [17]. As $N_{\mathrm{d} i}^{+}, N_{\mathrm{a} j}^{-}$depend not only on the densities and binding energies of deep centers, but also on $\Gamma, g_{\mathrm{c}}, g_{\mathrm{v}}$, the location of the Fermi level and the band carrier densities are largely dependent on the magnitude of PF. The ideal (but unachievable) situation of a compensated semiconductor assumed to be free from PF constitutes a limiting case which is also described by the above equations (4 to 11$)$ : when $\Gamma, g_{c}, g_{\mathrm{v}}$, tend to zero the carrier densities tend to $n_{00}, p_{00}$ and the ionization probability reduces to the classical value, given by equation (9) when $u=0$. Therefore, in interpreting the data, the general expressions (4-11) must be used, and only the comparison with experiment allows us to recognize if $\Gamma$ is equal to zero or not. On the contrary, the common procedure which consists to assume $a$ priori that $\Gamma=0$ is totally unjustified.

2.2 BULK CONDUCTIVITY AND PERMITTIVITY IN THE PRESENCE OF PF. - The lattice and the carriers in extended and in localized states take part in the electric polarisation and in the conductivity of the sample. Thus, the total bulk complex conductivity $\sigma_{\text {act }}+i \omega \varepsilon_{\text {act }}$ experimentally measured on a sample between ohmic contacts is the sum of the separate contributions $\sigma_{\mathrm{ac}}+i \omega \varepsilon_{\mathrm{ac}}$ of lattice and band carriers, and $\left(\sigma_{\mathrm{ac}}+i \omega \varepsilon_{\mathrm{ac}}\right)_{\mathrm{cent}}$ of deep centers :

$$
\begin{aligned}
\sigma_{\mathrm{act}}+i \omega \varepsilon_{\mathrm{act}}=\sigma_{\mathrm{ac}}+ & \left(\sigma_{\mathrm{ac}}\right)_{\mathrm{cent}}+ \\
& +i \omega\left(\varepsilon_{\mathrm{ac}}+\left(\varepsilon_{\mathrm{ac}}\right)_{\mathrm{cent}}\right) .
\end{aligned}
$$

i) Effect of lattice and band carriers : in extrinsic semiconductors, only one of the two quantities $g_{c}, g_{\mathrm{v}}$ has a real importance. In an n-type semiconductor for instance, $g_{\mathrm{c}}$ is fixed by the electon population accumulated in the deepest wells, but the exact value of $g_{\mathrm{v}}$ is unimportant because the electron density at $E_{\mathrm{c} 0}+g_{\mathrm{v}} \Gamma$ is negligible as soon as $g_{\mathrm{v}} \Gamma / k T \gg 1$. It is thus possible, in order to simplify the formulae, to assume that $g_{\mathrm{c}}$ and $g_{\mathrm{v}}$ have the same value $g$. Equations $(10,11)$ of reference [17] are generalized by also taking into account the band carriers in PF of maximum magnitude $g \Gamma$; thus :

$$
\begin{aligned}
\frac{\sigma_{\mathrm{ac}}}{\sigma_{00}}=\frac{2}{\sqrt{\Pi}} \int_{0}^{g}\left\{1+\alpha\left(\exp \left(\frac{2 u \Gamma}{k T}\right)-1\right) \frac{x(u) \exp \left(-\frac{u \Gamma}{k T}\right)+\omega^{2} \tau_{00}^{2}}{x^{2}(u)+\omega^{2} \tau_{00}^{2}}\right\} \exp \left(-u^{2}-\frac{u \Gamma}{k T}\right) \mathrm{d} u+ \\
+\frac{2}{\sqrt{\Pi}}\left\{\exp \left(-\frac{g \Gamma}{k T}\right)+2 \alpha \sinh \left(\frac{g \Gamma}{k T}\right) \frac{x(g) \exp \left(-\frac{g \Gamma}{k T}\right)+\omega^{2} \tau_{00}^{2}}{x^{2}(g)+\omega^{2} \tau_{00}^{2}}\right\} \int_{g}^{\infty} \exp \left(-u^{2}\right) \mathrm{d} u . \\
\frac{\varepsilon_{\mathrm{ac}}}{\varepsilon_{\mathrm{sc}}}=1+\frac{8 \alpha(1-\alpha)}{\sqrt{\Pi}}\left(\int_{0}^{g} \frac{\sin ^{2}(u \Gamma / k T)}{x^{2}(u)+\omega^{2} \tau_{00}^{2}} \exp \left(-u^{2}\right) \mathrm{d} u+\frac{\sin ^{2}(g \Gamma / k T)}{x^{2}(g)+\omega^{2} \tau_{00}^{2}} \int_{g}^{\infty} \exp \left(-u^{2}\right) \mathrm{d} u\right)
\end{aligned}
$$


where :

$$
\begin{aligned}
x(u) & =\alpha \exp (-u \Gamma / k T)+(1-\alpha) \exp (u \Gamma / k T) \\
\sigma_{00} & =q \mu_{\mathrm{n}} n_{00}(\mathrm{n} \text { type }), \text { or }: \sigma_{00}=q \mu_{\mathrm{p}} p_{00}(\mathrm{p} \text { type }) \\
\tau_{00} & =\varepsilon_{\mathrm{sc}} / \sigma_{00} .
\end{aligned}
$$

As $\omega \rightarrow \infty, \sigma_{\mathrm{ac}}$ tends to the limit :

$$
\sigma_{\infty}=\frac{2 \sigma_{00}}{\sqrt{\Pi}}\left(\int_{0}^{g} x(-u) \exp \left(-u^{2}\right) \mathrm{d} u+x(-g) \int_{g}^{\infty} \exp \left(-u^{2}\right) \mathrm{d} u\right) .
$$

As $\omega \rightarrow 0, \sigma_{\text {ac }}$ tends to the dc conductivity:

$$
\sigma_{\mathrm{dc}}=\frac{2 \sigma_{00}}{\sqrt{\Pi}}\left(\int_{0}^{g} \frac{\exp \left(-u^{2}\right)}{x(u)} \mathrm{d} u+\frac{1}{x(g)} \int_{g}^{\infty} \exp \left(-u^{2}\right) \mathrm{d} u\right) .
$$

At frequencies where $x(g) \exp (-g \Gamma / k T) \ll$ $\omega^{2} \tau_{00}^{2} \ll x^{2}(g)$, the last term in equation (13) varies as $\omega^{2}$. When this contribution (due to carriers in deepest wells) is predominant in the second member of equation (13), the measured values of $\sigma_{\text {ac }}$ increase as $\omega^{2}$ at high frequencies before saturating at $\sigma_{\infty}$. Abdalla and Pistoulet [33], in order to explain high frequency data on semi-insulating GaAs, introduced the assumption of fluctuations of defined magnitude superimposed to the Gaussian distribution. In fact, these fluctuations are likely constituted by the wells of maximum magnitude which were not taken into account in reference [33]. The $\sigma_{\mathrm{ac}} \propto \omega^{2}$ dependence reported for a-Si : $\mathrm{H}$ films, by Pistoulet et al. [34] and by Jonscher [35] in various materials, has likely the same origin. For the specific samples considered in the present paper the $\sigma_{\text {ac }} \propto \omega^{2}$ law is not observed. Besides, numerical calculations show that the conductivity due to carriers in wells of maximum magnitude remains small compared to the term corresponding to the Gaussian distribution up to the highest frequency $\left(10^{6} \mathrm{~Hz}\right)$ at which the measurements have been performed.

ii) Contribution of deep centers: deep centers contribute to the complex conductivity of a material if their ionization probability changes with the external field. In the case of microscopically homogeneous semiconductors, free from PF as commonly assumed, the local energy difference between impurity levels and Fermi level, in the bulk, or in samples between ohmic contacts, remains independent of the applied field ; consequently the degree of ionization of the centers is independent of the external field except in $p-n$ junctions, or in surface depletion or accumulation layers where the changes in band bending due to the external field change the ionization probability of the centers. Therefore only the centers located in these space charge layers contribute [36] to the complex admittance of the sample. Moreover the field enhancement of bulk conductivity is generally considered as due to SCLC, and this effect has been used for the determination of the density of midgap states in a-Si: $\mathrm{H}$.

The situation is quite different in samples exhibiting potential fluctuations because deep levels can cross the Fermi level not only near the surface, but also in a multitude of wells and hills in the bulk. Pistoulet and Abdalla [29] reported that, due to the image force barrier lowering, the maximum magnitude of PF is reduced by the application of an external field. Two consequences are following from that : i) field enhanced band conductivity results simply from image force barrier lowering of PF ; ii) the degree of ionization of deep centers now changes with the field, so that these centers can contribute to complex conductance, not only in surface layers but also in the bulk and in samples between ohmic contacts [17].

Therefore no reliable interpretation of compensated semiconductor transport data can be carried out without taking into account the existence and the field dependence of PF. As shown in reference [29], the maximum magnitude $g \Gamma$ of $P F$ varies with the external field $\varepsilon$ according to the law :

$$
g=g_{0}\left(1-\left(\mathcal{E} / \varepsilon_{\mathrm{c}}\right)^{1 / 2}\right)
$$

where $\varepsilon_{c}$ is a critical field characteristic of the sample. We are interested in compensated semiconductors containing a noticeable density of ionized centers, so that the potential wells and hills created by individual centers overlap each other, resulting in relatively smooth variations as shown in figure 1. Then an external field produces statistically equal and opposite shifts of the opposite walls of any well and hill, leaving the Gaussian distribution $P_{\mathrm{G}}\left(E_{\mathrm{c}}\right)$ unchanged. This explains why only the maximum magnitude $g_{\mathrm{c}} \Gamma$ of wells and $g_{\mathrm{v}} \Gamma$ of hills is changed by the field, as confirmed by experiment (see below). In many cases, the Fermi level crosses the levels of the main species of centers present in the 
material either in the wells as illustrated in figure 8 or in the hills. In the first case for instance, only the knowledge of $g_{\mathrm{c}}$ is significant for determining the average degree of ionization of the centers ; therefore the exact value of $g_{\mathrm{v}}$ is unimportant, and may be assumed to be equal to $g_{\mathrm{c}}$. Then in the following we shall again assume $g_{\mathrm{c}}=g_{\mathrm{v}}=g$ in equation (11).

When the external field varies from zero to $\mathcal{E}, g$ goes from $g_{0}$ to $g$, and the charge density of ionized centers changes by the amount :

$$
\begin{aligned}
\Delta \rho=q\left\{\sum _ { i } \left(N_{\mathrm{d} i}^{+}(g)\right.\right. & \left.-N_{\mathrm{d} i}^{+}\left(g_{0}\right)\right)- \\
& \left.-\sum_{j}\left(N_{\mathrm{a} j}^{-}(g)-N_{\mathrm{a} j}^{-}\left(g_{0}\right)\right)\right\} .
\end{aligned}
$$

When a small low frequency field is applied, the corresponding variation $\mathrm{d} \rho$ of the average charge density of ionized centers is therefore equal to :

$$
\mathrm{d} \rho=q\left\{\sum_{i} \frac{\partial N_{\mathrm{d} i}^{+}}{\partial g}-\sum_{j} \frac{\partial N_{\mathrm{aj}}^{-}}{\partial g}\right\} \mathrm{d} g
$$

As the frequency of the external field is increased, deep centers have not enough time to reach equilibrium, and the local densities of ionized centers obey the rate equations :

$$
\begin{aligned}
& \frac{\partial N_{\mathrm{d} i}^{+}(u)}{\partial t}=-\frac{N_{\mathrm{d} i}^{+}(u)-N_{\mathrm{d} i 0}^{+}(u)}{\tau_{i}} ; \\
& \frac{\partial N_{\mathrm{a} j}^{-}(u)}{\partial t}=-\frac{N_{\mathrm{a} j}^{-}(u)-N_{\mathrm{a} j 0}^{-}(u)}{\tau_{j},}
\end{aligned}
$$

where $N_{\mathrm{d} i 0}^{+}(u), N_{\mathrm{aj} 0}^{-}(u)$ are local equilibrium densities at zero field, and $\tau_{i}, \tau_{j}$ are time constants characteristic of the combined emission-capture process. The complex amplitudes $\overline{N_{\mathrm{d} i}^{+}}, \overline{N_{\mathrm{aj}}^{-}}$at angular frequency $\omega$ are thus :

$$
\overline{N_{\mathrm{d} i}^{+}}=\frac{\overline{N_{\mathrm{d} i 0}^{+}}}{1+i \omega \tau_{i}} ; \quad \overline{N_{\mathrm{aj}}^{-}}=\frac{\overline{N_{\mathrm{a} j 0}^{-}}}{1+i \omega \tau_{j}} .
$$

According to equation (22) the small signal ac complex amplitude $\overline{\mathrm{d} \rho}$ of the center charge density has the following expression :

$$
\overline{\mathrm{d} \rho}=q\left(\sum_{i} \frac{1-i \omega \tau_{i}}{1+\omega^{2} \tau_{i}^{2}} \cdot \frac{\partial N_{\mathrm{d} i}^{+}}{\partial g}-\sum_{j} \frac{1-i \omega \tau_{j}}{1+\omega^{2} \tau_{j}^{2}} \cdot \frac{\partial N_{\mathrm{a} j}^{-}}{\partial g}\right) \overline{\mathrm{d} g}
$$

where $\overline{\mathrm{d} g}=(\partial g / \partial \mathcal{E}) \overline{\mathrm{d}}$. The quantity $\partial g / \partial \mathcal{E}$ can be obtained from dc conductivity versus field data using (Eq. 20). The derivative $\partial N_{\mathrm{d} i}^{+} / \partial g$ (and similarly $\partial N_{\mathrm{aj}}^{-} / \partial g$ ) is calculated from equation (11). Neglecting the very small variations of $E_{\mathrm{F}}$ and $n_{00}$, we obtain :

$$
\frac{1}{N_{\mathrm{d} i}} \frac{\partial N_{\mathrm{d} i}^{+}}{\partial g}=-\frac{2}{\sqrt{\Pi}} \frac{\Gamma}{k T}\left(\alpha \frac{M(g)}{(1+M(g))^{2}}-(1-\alpha) \frac{M(-g)}{(1+M(-g))^{2}}\right) \int_{g}^{\infty} \exp \left(-u^{2}\right) \mathrm{d} u
$$

and a similar expression for $\frac{1}{N_{\mathrm{a} j}} \frac{\partial N_{\mathrm{a} j}^{-}}{\partial g}$.

The current density, due to centers, entering a cylindrical sample of length $L$, is the time derivative of the charge contained in the sample; so the complex amplitude of the current density is :

$$
\overline{J_{\text {cent }}}=i \omega L \overline{\mathrm{d} \rho} \text {. }
$$

According to equations $(25,27)$, the contribution of the centers to the conductivity and to the permittivity of the sample may be expressed as :

$$
\begin{aligned}
\sigma_{\mathrm{ac} \text { cent }} & =\omega q L\left(\sum_{i} \frac{\omega \tau_{i}}{1+\omega^{2} \tau_{i}^{2}} \cdot \frac{\partial N_{\mathrm{d} i}^{+}}{\partial g}-\sum_{j} \frac{\omega \tau_{j}}{1+\omega^{2} \tau_{j}^{2}} \cdot \frac{\partial N_{\mathrm{a} j}^{-}}{\partial g}\right) \frac{\partial g}{\partial \mathcal{E}} \\
\varepsilon_{\text {ac cent }} & =q L\left(\sum_{i} \frac{1}{1+\omega^{2} \tau_{i}^{2}} \cdot \frac{\partial N_{\mathrm{d} i}^{+}}{\partial g}-\sum_{j} \frac{1}{1+\omega^{2} \tau_{j}^{2}} \cdot \frac{\partial N_{\mathrm{a} j}^{-}}{\partial g}\right) \frac{\partial g}{\partial \mathcal{E}} .
\end{aligned}
$$

The expressions of $\partial N_{\mathrm{d} i}^{+} / \partial g$ and of $-\partial N_{\mathrm{aj}}^{-} / \partial g$ obtained from the relations (11) are quite similar. Thus the knowledge of $\sigma_{\mathrm{ac} \text { cent }}$ and $\varepsilon_{\mathrm{ac} \text { cent }}$ alone does not allow us to distinguish if the peaks correspond to donor or acceptor states.

When $\omega$ goes from zero to infinity, the variation of $\varepsilon_{\text {ac cent }}$ deduced from equation (29) is equal to :

$$
\left(\varepsilon_{\mathrm{LF}}-\varepsilon_{\mathrm{HF}}\right)_{\mathrm{cent}}=q L\left(\sum_{i} \frac{\partial N_{\mathrm{d} i}^{+}}{\partial g}-\sum_{j} \frac{\partial N_{\mathrm{a} j}^{-}}{\partial g}\right) \frac{\partial g}{\partial \mathcal{E}} .
$$


The total variation of the permittivity results from equations $(14,30)$ :

$$
\begin{array}{r}
\left(\varepsilon_{\mathrm{LF}}-\varepsilon_{\mathrm{HF}}\right)_{t}=\varepsilon_{\mathrm{sc}} \frac{8 \alpha(1-\alpha)}{\sqrt{\Pi}}\left(\int_{0}^{g} \frac{\sinh ^{2}(u \Gamma / k T)}{x^{2}(u)} \exp \left(-u^{2}\right) \mathrm{d} u+\frac{\sin ^{2}(g \Gamma / k T)}{x^{2}(g)} \int_{g}^{\infty} \exp \left(-u^{2}\right) \mathrm{d} u\right)+ \\
+\left(\varepsilon_{\mathrm{LF}}-\varepsilon_{\mathrm{HF}}\right)_{\mathrm{cent}} .
\end{array}
$$

In a-Si: $\mathrm{H}$ samples, the average density of localized levels crossing the Fermi level is significant, so the contribution of band carriers to $\left(\varepsilon_{\mathrm{LF}}-\varepsilon_{\mathrm{HF}}\right)_{\mathrm{t}}$ is generally small compared to that of localized states, and the second member of equation (31) reduces nearly to $\left(\varepsilon_{\mathrm{LF}}-\varepsilon_{\mathrm{HF}}\right)_{\text {cent }}$. Using equations $(13,14$, 28,29 ) it is thus possible, by fitting complex conductivity data versus frequency and temperature, to evaluate separately the densities of localized states and of band carriers. This is a fundamental advantage over other methods like FE and sweep-out.

In summary, in the case of samples between ohmic contacts :

- The experimental observation of large variations $\left(\varepsilon_{\mathrm{LF}}-\varepsilon_{\mathrm{HF}}\right)_{\mathrm{t}}$ of $\varepsilon_{\text {act }}$ between low and high

$$
\begin{aligned}
Q_{\mathrm{sc}}= \pm\left(\begin{array}{ll}
2 & \varepsilon_{\mathrm{sc}} q
\end{array}\right)^{1 / 2} \mid \sum_{i, j} \int_{0}^{\psi_{\mathrm{s}}} \Delta\left(N_{\mathrm{d} i}^{+}-N_{\mathrm{aj}}^{-}\right) \mathrm{d} \psi-n\left(\frac{k T}{q}\left(\exp \frac{q \psi_{\mathrm{s}}}{k T}-1\right)-\psi_{\mathrm{s}}\right)- \\
\quad-\left.p\left\{\frac{k T}{q}\left(\exp \left(-\frac{q \psi_{\mathrm{s}}}{k T}\right)-1\right)+\psi_{\mathrm{s}}\right\}\right|^{1 / 2}
\end{aligned}
$$

where $\Delta N_{\mathrm{d} i}^{+}, \Delta N_{\mathrm{a} j}^{-}$are the variations of $N_{\mathrm{d} i}^{+}$, $N_{\mathrm{aj}}^{-}$, caused by a variation $-q \psi$ of $E_{\mathrm{co}}-E_{\mathrm{F}}$. In the case of donors for instance :

$$
\begin{aligned}
& \Delta N_{\mathrm{d} i}^{+}(\psi)= \\
& =\int_{-g}^{g}\left(N_{\mathrm{d} i}^{+}\left(u-\frac{q \psi}{\Gamma}\right)-N_{\mathrm{d} i}^{+}(u)\right) P(u) \mathrm{d} u
\end{aligned}
$$

where $g$ is a function of $\mathrm{d} \psi / d x$.

Measurement of the differential surface capacitance $C_{\mathrm{D}}\left(\psi_{0}\right)$ is performed by superposing a low amplitude ac surface potential to the dc potential $\psi_{0}$ : frequencies constitutes an evidence of the existence of PF and of the presence of deep centers.

- A new method for the determination of the densities of deep centers is proposed. Provided that the gap state density consists of a limited number of peaks well separated in energy, this method allows us to determine separately the carrier density and the densities of the different species of centers.

2.3 SURFACE LAYER CAPACITANCE. - In field effect experiments, the quantity generally measured is the capacitance of a MIS diode, or of a rectifying Schottky barrier. In the presence of PF the measured surface charge density is an average over the surface of the sample. It was shown [24] that, if $\psi_{\mathrm{s}}$ is the average surface potential, the net charge density can be expressed as follows:

$$
\psi_{\mathrm{s}}(t)=\psi_{0}+\psi_{1} \exp (i \omega t), \text { with } \quad \psi_{1} \ll \psi_{0}
$$

At very low frequencies, the degree of ionization of deep centers adjusts instantaneously to the potential changes imposed by the external source, so that $Q_{\mathrm{sc}}(t)$ is a function of $\psi_{\mathrm{s}}(t)$ only. The differential capacitance at very low frequencies is thus expressed as :

$$
C_{\mathrm{DLF}}=\left|\frac{\partial Q_{\mathrm{sc}}}{\partial \psi_{\mathrm{s}}}\right|
$$

or, according to equation (32) :

$$
C_{\mathrm{DLF}}=\frac{q \varepsilon_{\mathrm{sc}}}{Q_{\mathrm{sc}}}\left\{\sum_{i, j} \Delta\left(N_{\mathrm{d} i}^{+}\left(\psi_{\mathrm{s}}\right)-N_{\mathrm{aj}}^{-}\left(\psi_{\mathrm{s}}\right)\right)-n\left(\exp \frac{q \psi_{\mathrm{s}}}{k T}-1\right)+p\left(\exp \left(-\frac{q \psi_{\mathrm{s}}}{k T}\right)-1\right)\right\} .
$$

This expression shows that both deep centers and free carriers contribute to $C_{\mathrm{DLF}}$. In the presence of $\mathrm{PF}$, the total carrier densities $n$ and $p$ are larger by a factor $\sigma_{\infty} / \sigma_{\mathrm{dc}}$ than the densities $n_{\mathrm{f}}, p_{\mathrm{f}}$ deduced from dc measurements. So, if $g \Gamma / k T \gg 1$, the contribution of free carriers to $C_{\mathrm{D}}$ may become predominant in equation (36), even if $\sigma_{\mathrm{dc}}$ is very small. If, as in most papers, this contribution of band carriers is 
disregarded, field effect determinations of gap state density are overevaluated, particularly when approaching the band edges. This is likely the reason for the similarity between the gap state density determined in a-Si : $\mathrm{H}$ by FE measurements, and the well known surface charge density of band carriers versus $\psi_{0}$ in ideal MIS diodes [37]. When the signal frequency is increased, the local density of ionized impurities can no longer follow the potential variations, so that $Q_{\mathrm{sc}}(\psi, t)$ depends not only on the instantaneous value $\psi(t)$ of the local potential, but also on time, i.e. on the frequency of the ac signal. The differential capacitance seen from the terminals of the device is then :

$$
\begin{aligned}
C_{\mathrm{D}}\left(\psi_{0}\right) & =\left|\left(\mathrm{d} Q_{\mathrm{sc}} / \mathrm{d} t\right) /\left(\mathrm{d} \psi_{\mathrm{s}} / \mathrm{d} t\right)\right| \\
& =\left|\frac{\partial Q_{\mathrm{sc}}}{\partial \psi_{\mathrm{s}}}+\left(\frac{\partial Q_{\mathrm{sc}}}{\partial t}\right) /\left(\frac{\mathrm{d} \psi_{\mathrm{s}}}{\mathrm{d} t}\right)\right| .
\end{aligned}
$$

In this expression, only the spectral components at angular frequency $\omega$ are taken into account. The quantity $\Delta N_{\mathrm{d} i}^{+}$(and similarly $\Delta N_{\mathrm{aj}}^{-}$) in equation (32), is now the sum of a term $\Delta N_{\mathrm{d} i}^{+}(\psi)$ depending only on $\psi(t)$ and of a term $\Delta N_{\mathrm{d} i}^{+}(\psi, t)-\Delta N_{\mathrm{d} i 0}^{+}(\psi)$ depending on frequency due to the finite emission rate of the centers, where $\Delta N_{\mathrm{d} i 0}^{+}(\psi)$ is the limit of $\Delta N_{\mathrm{d} i}^{+}(\psi, t)$ when $\omega \rightarrow 0$. Therefore the expression of $\Delta N_{\mathrm{d} i}^{+}$in equation (32) is :

$$
\begin{aligned}
& \Delta N_{\mathrm{d} i}^{+}=\Delta N_{\mathrm{d} i}^{+}(\psi)+ \\
& +\left(\Delta N_{\mathrm{d} i}^{+}(\psi, t)-\Delta N_{\mathrm{d} i 0}^{+}(\psi)\right)
\end{aligned}
$$

The complex amplitude of the quantity in brackets is obtained from the rate equations $(23,24)$ :

$$
\begin{aligned}
\overline{\Delta N_{\mathrm{d} i}^{+}(\psi, t)}-\overline{\Delta N_{\mathrm{d} i 0}^{+}(\psi)} & = \\
= & -\frac{i \omega \tau_{i}}{1+i \omega \tau_{i}} \overline{\Delta N_{\mathrm{d} i 0}^{+}(\psi)} .
\end{aligned}
$$

According to equations $(32,33,36,39)$ the contribution of donor species $i$ to the complex capacitance of the sample is :

$$
C_{\mathrm{i}}+\frac{G_{i}}{i \omega}=\frac{q \varepsilon_{\mathrm{sc}}}{Q_{\mathrm{sc}}\left(\psi_{0}\right)}\left(\Delta N_{\mathrm{d} i}^{+}\left(\psi_{0}\right)-\frac{1}{\overline{\psi_{1}}} \frac{i \omega \tau_{i}}{1+i \omega \tau_{i}} \int_{0}^{\psi_{0}} \overline{\Delta N_{\mathrm{d} i 0}^{+}(\psi)} \mathrm{d} \psi\right)
$$

and a similar expression for the contribution of acceptor centers. Therefore the complex capacitance $C_{\mathrm{D}}+G_{\mathrm{D}} / i \omega$ of the surface layer submitted to a dc surface potential $\psi_{0}$, can be written :

$$
\begin{gathered}
C_{\mathrm{D}}\left(\psi_{0}, \omega\right)+\frac{G_{\mathrm{D}}\left(\psi_{0}, \omega\right)}{i \omega}=C_{\mathrm{c}}+\sum_{i} C_{i}+\sum_{j} C_{j}+ \\
+\frac{1}{i \omega}\left(\sum_{i} G_{i}+\sum_{j} G_{j}+G_{\mathrm{c}}\right)
\end{gathered}
$$

where $C_{\mathrm{c}}+\frac{G_{\mathrm{c}}}{i \omega}$ is the contribution of lattice and band carriers. Introducing the low and high frequency limits $C_{i \mathrm{LF}}$ and $C_{i \infty}$ of $C_{i}$ in equation (40), $C_{i}(\omega)$ and $G_{i}(\omega)$ can be expressed as :

$$
\begin{aligned}
C_{i} & =C_{i \infty}+\frac{C_{i \mathrm{LF}}-C_{i \infty}}{1+\omega^{2} \tau_{i}^{2}} \\
G_{i} & =\frac{\omega^{2} \tau_{i}}{1+\omega^{2} \tau_{i}^{2}}\left(C_{i \mathrm{LF}}-C_{i \infty}\right) .
\end{aligned}
$$

Thus $C_{\mathrm{D}}$ and $G_{\mathrm{D}}$ depend in a complicated way not only on the surface potential, center density and binding energy, total band carrier density, frequency, temperature, but also on $\Gamma$ and $g$.

\footnotetext{
JOURNAL DE PHYSIQUE. - T. 49, N 11, NOVEMBRE 1988
}

\section{Experimental data and discussion.}

3.1 Procedure. - The determination of the physical quantities $g, \Gamma, E_{\mathrm{F}}-E_{\mathrm{c} 0}, N_{\mathrm{d} i}, N_{\mathrm{aj}}$ is based on the comparison of the variations of $\sigma_{\text {act }}(\omega, T)+$ $i \omega \varepsilon_{\text {act }}(\omega, T)$ calculated by the previous theory, with the experimental data. First of all, it is necessary to be sure of the ohmicity of the contacts. The absence of a contact barrier at the metal-a-Si : $\mathrm{H}$ interface is proved by the fact that, in our samples, the I(V) curves do not depend on the voltage polarity, as shown by systematic measurements. The possible existence of a contact resistance in series with the impedance of the amorphous layer must also be excluded because, in this case, the measured capacitance should decrease as $\omega^{-2}$ at high frequencies, in contradiction with the experimental data. So the non-linearity of the I(V) curves results very likely from the field enhancement of the conductivity of the amorphous material itself.

In order to determine the zero field value $g_{0}$ of $g$, the first step consists in determining $g_{0}(T)$ and $\Gamma$ by fitting the observed temperature dependence of $\sigma_{\mathrm{dc}} / \sigma_{\infty}$ at low fields with the aid of equations $(18,19)$. The parameter $\alpha$ which changes the location of the $\sigma_{\mathrm{ac}}(\omega)$ isotherms is detemined in successive steps. As we shall see below, $\sigma_{\infty}$ is sometimes directly measured. For given values of 
$g_{0}$ and $\Gamma / k T$, equations $(7,16,18)$ relate $\mu_{\mathrm{n}}$ and $\left(E_{\mathrm{F}}-E_{\mathrm{c} 0}\right) / k T$. The value of $\mu_{\mathrm{n}}$ is adjusted in order to reach the correct location of the Fermi level relative to the deep levels. As $\left(\sigma_{\mathrm{ac}}\right)_{\mathrm{cent}}$ and $\left(\varepsilon_{\mathrm{ac}}\right)_{\mathrm{cent}}$ are very sensitive to $E_{c 0}-E_{\mathrm{F}}$, the correct value of $\mu_{\mathrm{n}}$ is the one which allows us to fit the data, and therefore the method also leads to the determination of the mobility of band carriers.

When $\Gamma$ is large $\sigma_{\infty}$ may not be reached at the highest frequency of experiment. At constant Fermi energy, $\sigma_{\infty}$ and $\mu_{n}$ are proportional. However changing $\sigma_{\infty}$ also changes $\tau_{00}$ (Eqs. 17, 18) and $\sigma_{\mathrm{ac}}(\omega, T)$ due to band carriers (Eq. 13). Therefore by successive approximations, the correct value of $\sigma_{\infty}$ is obtained from the fit, with an uncertainty which, in the worst case, is less than one order of magnitude, as already explained in reference [17]. The localized gap states are responsible for the bumps observed on the $\sigma_{\text {act }}(\omega)$ isotherms, and for the large variations of $\varepsilon_{\text {act }}(\omega)$. According to equation (28), the isotherms $\left(\sigma_{\mathrm{ac}}\right)_{\mathrm{cent}} / \omega$ (and roughly the isotherms $\sigma_{\text {act }} / \omega$ ) must go through a series of maxima at angular frequencies $1 / \tau_{i}$ (or $1 / \tau_{j}$ ) related to the various species of centers, as is observed for instance in figure 5 in the case of undoped a-Si: H. For each species, the binding energy $E_{\mathrm{d} i}$ and the high temperature limit $\tau_{i 0}$ of $\tau_{i}$ are deduced from the Arrhenius plot of $\tau_{i}$ versus $1 / T$ :

$$
\tau_{i}=\tau_{i 0} \exp \left(E_{\mathrm{d} i} / k T\right)
$$

if the activation energy of the capture cross section is neglected. Then the unknowns concerning the localized states reduce to the densities $N_{\mathrm{d} i}$ (or $\left.N_{\text {aj }}\right)$. These densities are obtained by fitting the experimental $\sigma_{\text {act }}(\omega, T)$ and $\varepsilon_{\text {act }}(\omega, T)$ data. Because the contribution of deep centers is very sensitive to the location of the Fermi level relatively to the bottom of the deepest wells, very small changes in $E_{\mathrm{d} i}, \sigma_{\infty}, \mu_{\mathrm{n}}, g \Gamma$ lead generally to large changes in the frequency and temperature dependences of $\left(\sigma_{\mathrm{ac}}\right)_{\mathrm{cent}}$ and $\left(\varepsilon_{\mathrm{ac}}\right)_{\mathrm{cent}}$. Consequently the values of the quantities $E_{\mathrm{d} i}, N_{\mathrm{d} i}, \tau_{i 0}$ are refined by successive approximations until the best fit of the data is obtained. Moreover, the band carrier densities and the densities $N_{\mathrm{d} i}^{+}, N_{\mathrm{a} j}^{-}$of ionized deep centers must satisfy the average neutrality equation (6).

3.2 EXPERIMENTAL DATA AND DETERMINATION OF THE PHYSICAL PARAMETERS. - The a-Si films, supplied by Kaplan at Thomson-CSF, were grown by CVD on. heavily doped silicon single crystal substrates. The mehods of deposition and doping have been reported by Sol et al. [38] and Magariño et $a l$. [39]. The data reported in this paper refer to two kinds of deposited samples, without post hydrogenation :

— undoped films, $3 \mu \mathrm{m}$ thick
- boron-doped films : $1.1 \mu \mathrm{m}$ thick, with a doping ratio in the gas phase $\left(\mathrm{B}_{2} \mathrm{H}_{6}\right) /\left(\mathrm{SiH}_{4}\right)$ equal to $10^{-3}$.

For complex conductance measurements, the metallic ohmic contact of the sandwich was made by evaporating successively (after etching) a $200 \AA$ thick titanium film covered by an aluminium electrode of $2000 \AA$. The area of the dots was 1.77 $\mathrm{mm}^{2}$. The dc measurements were carried out using a 225 Keithley current source and a 616 Keithley electrometer. The ac complex permittivity measurements were performed, from $10^{-2} \mathrm{~Hz}$ to $10^{6} \mathrm{~Hz}$, with a frequency response analyser 1174 Schlumberger associated with a desktop computer HP $9825 \mathrm{~A}$. Between $50 \mathrm{~Hz}$ and $10^{5} \mathrm{~Hz}$, the accuracy of the method was confirmed by measurements made with an admittance bridge GENERAL RADIO $1615 \mathrm{~A}$ associated with a lock-in amplifier PAR 124 A. It was checked that the samples corresponding to different dots evaporated on the same film had similar characteristics, but the data reported below correspond to individual undoped or doped samples.

i) Undoped $n$ type sample $:$ it is shown below that the magnitude of PF is large in this material, so the dc conductance becomes very small at low tempera-

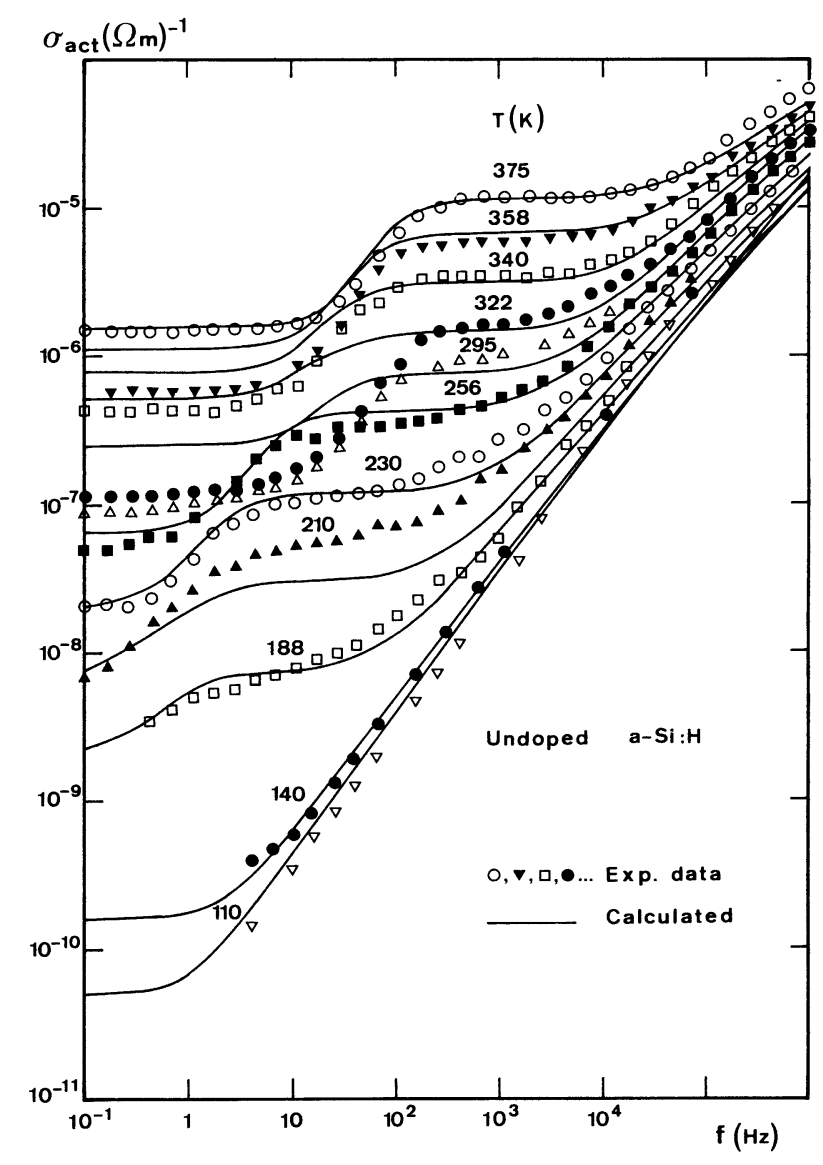

Fig. 2. - Conductivity $\sigma_{\text {act }}(T)$ versus frequency of undoped a-Si:H : $(\mathrm{O}, \boldsymbol{\nabla}, \square \ldots)$ Experiment ; $(\longrightarrow)$ Calculated values (Eqs. $(12,13,28)$ ). 
tures. Consequently, dc measurements could not be performed below $210 \mathrm{~K}$. Yet the range of ac measurements extended between $110 \mathrm{~K}$ and $375 \mathrm{~K}$. Experimental values of $\sigma_{\text {act }}$ and $\varepsilon_{\text {act }} / \varepsilon_{0}$ are plotted versus frequency in figures 2 and 3 . The saturation of

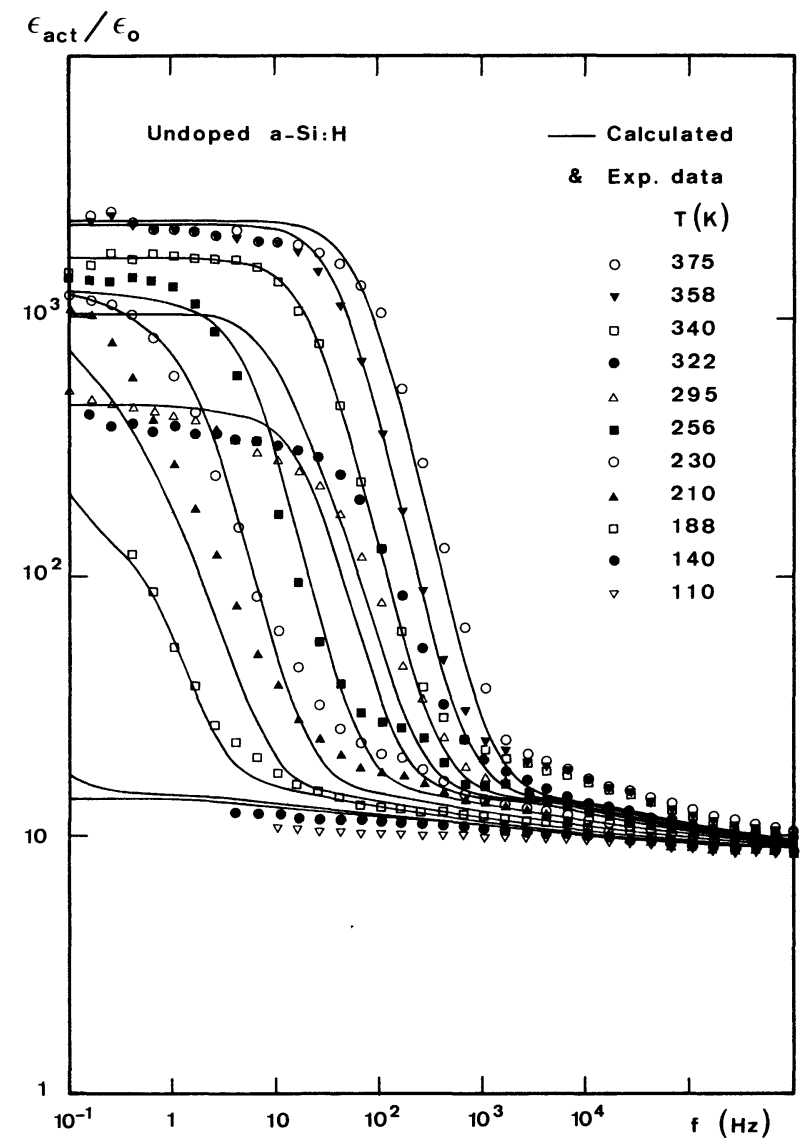

Fig. 3. - Relative permittivity $\varepsilon_{\text {act }}(T) / \varepsilon_{0}$ versus frequency of undoped a-Si : $\mathrm{H}:(O, \nabla, \square \ldots)$ Experimental data. $(-)$ Calculated values (Eqs. $(12,14,29)$ ).

the $\sigma_{\text {act }}(\omega)$ isotherms is not yet observed at the upper frequency of measurements, so $\sigma_{\infty}(T)$ is not directly known. As has been explained, the value which allows us to fit all the data is obtained by successive approximations, and is equal to $\sigma_{\infty}=$ $1.15 \times 10^{-4}(\Omega \mathrm{m})^{-1}$, nearly independent of temperature. By the same procedure we obtain : $\Gamma=0.137 \mathrm{eV}, \alpha=0.454$. At low temperatures, $\sigma_{\infty} / \sigma_{00}$ (Eq. 18) is roughly proportional to $\exp (g \Gamma / k T)$ so that, as $\sigma_{\infty} \simeq$ Const., $g \Gamma$ and $E_{\mathrm{c} 0}-E_{\mathrm{F}}$ are nearly proportional to temperature [17]. From the fit of $\sigma_{\mathrm{dc}}(T)$ and $\sigma_{\mathrm{ac}}(\omega, T)$ we find that, below $170 \mathrm{~K}, g_{0}=1.07 \times 10^{-2} T$. Above $170 \mathrm{~K}$, $g_{0}$ is constant and equal to 1.805 . The electron mobility allowing us to fit the data (as explained previously) is equal to $\mu_{\mathrm{n}}=10^{-4} \mathrm{~m}^{2} /$ V.s., approximately independent of temperature between 110 and $375 \mathrm{~K}$. Figure 4 shows the calculated isotherms $\sigma_{\mathrm{ac}}(\omega)$ corresponding to the band carrier conduc-

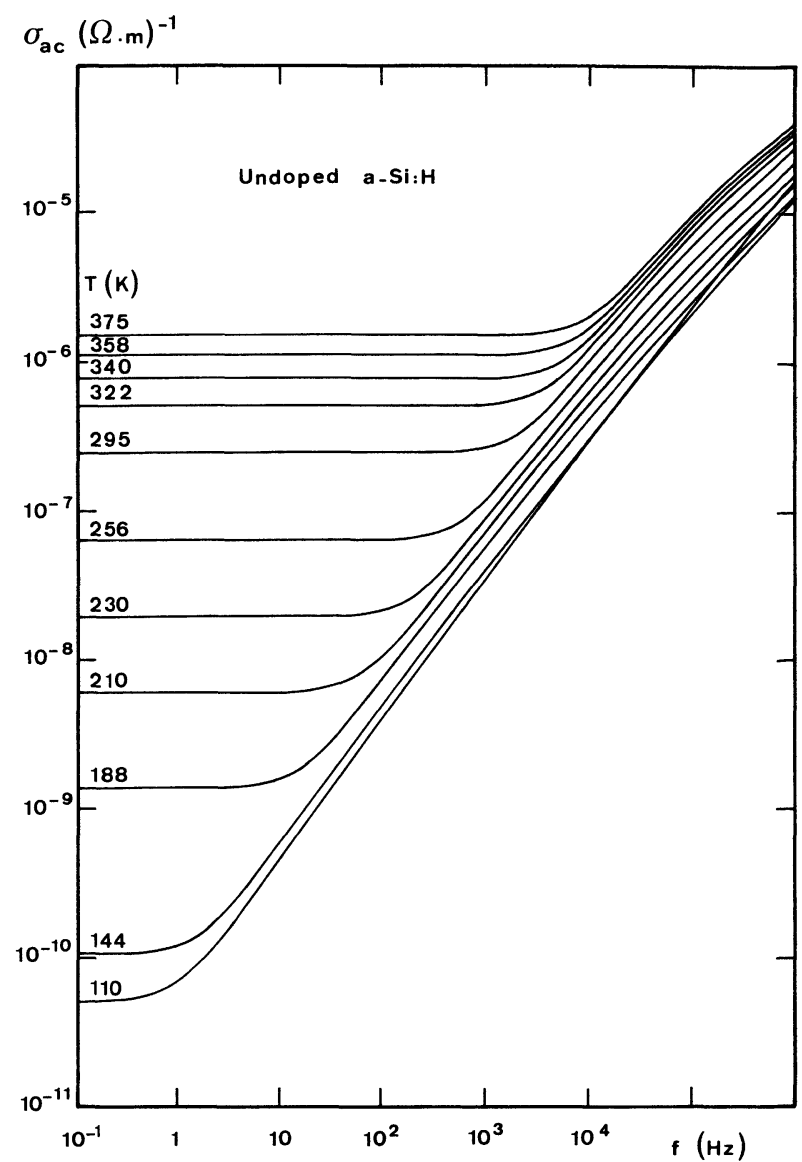

Fig. 4. - Calculated band carrier conductivity $\sigma_{\mathrm{ac}}(T)$ of undoped a-Si : $\mathrm{H}$ versus frequency (Eq. (13)).

tivity alone. The critical field $\varepsilon_{\mathrm{c}}$ is obtained by fitting the dependence of $\sigma_{\mathrm{dc}}(T)$ versus field with the aid of equation (20); the method is detailed below in the case of the doped sample. For the undoped sample we find $\varepsilon_{\mathrm{c}} \simeq 1.8 \times 10^{6} \mathrm{~V} / \mathrm{m}$, nearly independent of temperature. Then $\partial g / \partial \varepsilon$ is calculated (Eq. 20), for $\mathcal{E}=2 \times 10^{4} \mathrm{~V} / \mathrm{m}$ value of the externally applied field.

The existence of a discrete series of peaks in the gap state density is brought to the fore by plotting experimental $\sigma_{\text {act }} / \varepsilon_{\text {act }} \omega$ data versus $\omega$ at various temperature (Fig. 5). The curves exhibit a series of maxima corresponding roughly to the angular frequencies $1 / \tau_{i}$ at which $\left(\sigma_{\mathrm{ac}}\right)_{\text {cent }} / \varepsilon_{0} \omega$ is maximum (Eq. 28). For instance, the maxima observed at 340, $358,375 \mathrm{~K}$ correspond to the same center (or peak in the density of states). In order to determine $\tau_{i}(T)$ more precisely, the contribution of free carriers $\sigma_{\mathrm{ac}}(\omega, T)$ (Fig. 4) is subtracted from $\sigma_{\mathrm{act}}$, leading to the experimental values of $\left(\sigma_{\mathrm{ac}}\right)_{\text {cent }}$. Then the isotherms $\left(\sigma_{\mathrm{ac}}\right)_{\text {cent }} / \varepsilon_{0} \omega$ are plotted versus $\omega$ (Fig. 6), in the frequency range including the maxima. The binding energy $E_{\mathrm{d} 4} \simeq 0.37 \mathrm{eV}$ of this center, and $\tau_{40} \simeq 2 \times 10^{-8} \mathrm{~s}$ are deduced from the Arrhenius plot of $\tau_{4}$ versus $1 / T$ (insert of Fig. 6). The process is then repeated for the other maxima of the curves of 


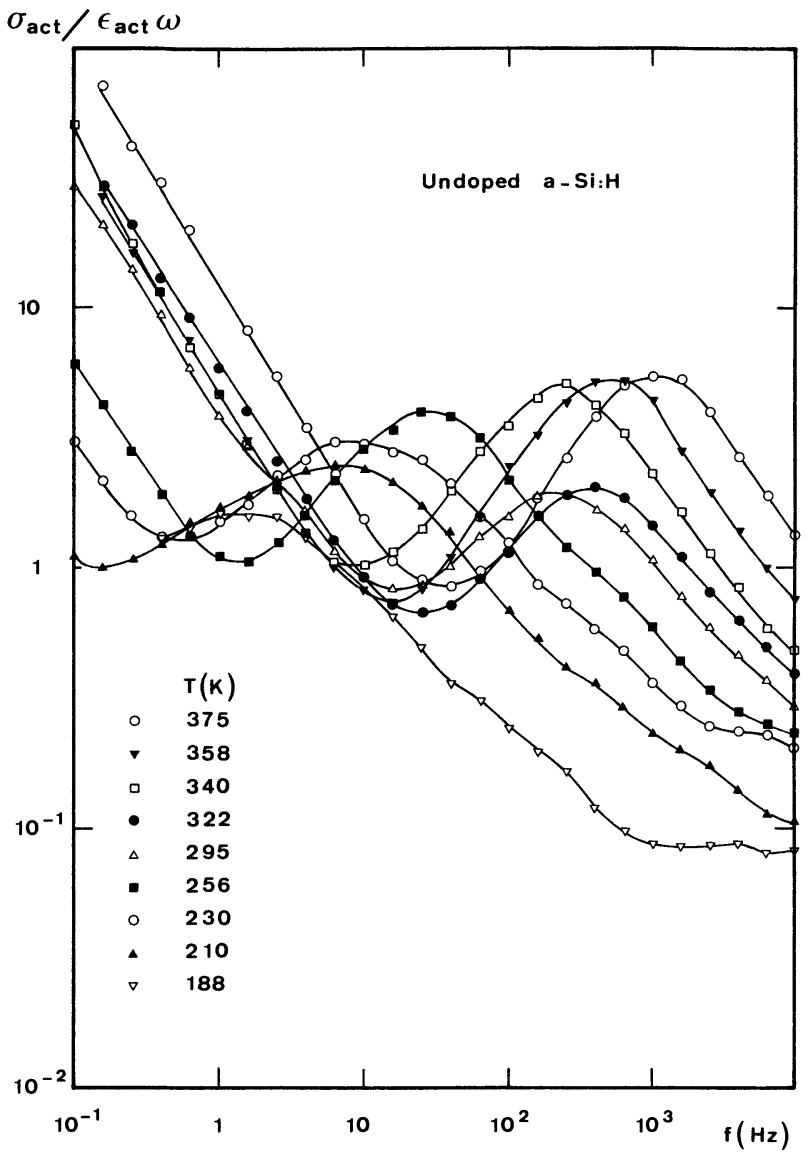

Fig. 5. - Experimental $\sigma_{\text {act }} / \varepsilon_{\text {act }} \omega$ data versus frequency at various temperatures (undoped a-Si $: \mathrm{H}$ ).

figure 5. However, particularly in undoped samples, some maxima include the contributions of more than one center; this leads to some uncertainty on the binding energy of centers. As $\left(\varepsilon_{\mathrm{LF}}-\varepsilon_{\mathrm{HF}}\right)_{\text {cent }}$ is very sensitive to minor changes of the parameters, it is then convenient to calculate the separate contributions of the centers to $\left(\varepsilon_{\mathrm{LF}}-\varepsilon_{\mathrm{HF}}\right)_{\mathrm{cent}}$, and to adjust $E_{\mathrm{d} i}, N_{\mathrm{d} i}, \tau_{i 0}$ by successive approximations until a good agreement with the data is approached. These values are again refined in order to obtain the best fit between the calculated and the experimental values of $\sigma_{\text {act }}(\omega, T)$ and $\varepsilon_{\text {act }}(\omega, T)$ in the whole ranges of temperatures and frequencies. The calculated curves and the experimental data are compared in figures $(2,3)$ for $\sigma_{\text {act }}$ and $\varepsilon_{\text {act }} / \varepsilon_{0}$. The calculations were made using the following characteristics of the four levels observed in the temperature range of experiments (as the donor or acceptor type of centers is unknown, the notation $E_{\mathrm{t}}, N_{\mathrm{t}}$ is used in place of $E_{\mathrm{d}}, N_{\mathrm{d}}$ or $E_{\mathrm{a}}, N_{\mathrm{a}}$ ):

$$
\begin{array}{ll}
E_{\mathrm{t} 1}=0.16 \mathrm{eV} ; & \tau_{10}=1 \times 10^{-5} \mathrm{~s} ; \\
E_{\mathrm{t} 2}=0.18 \mathrm{eV} ; & \tau_{20}=5.6 \times 10^{-5} \mathrm{~s} ; \\
E_{\mathrm{t} 3}=0.225 \mathrm{eV} ; & \tau_{30}=1 \times 10^{-6} \mathrm{~s} ; \\
E_{\mathrm{t} 4}=0.375 \mathrm{eV} ; & \tau_{40}=1.9 \times 10^{-8} \mathrm{~s} ;
\end{array}
$$

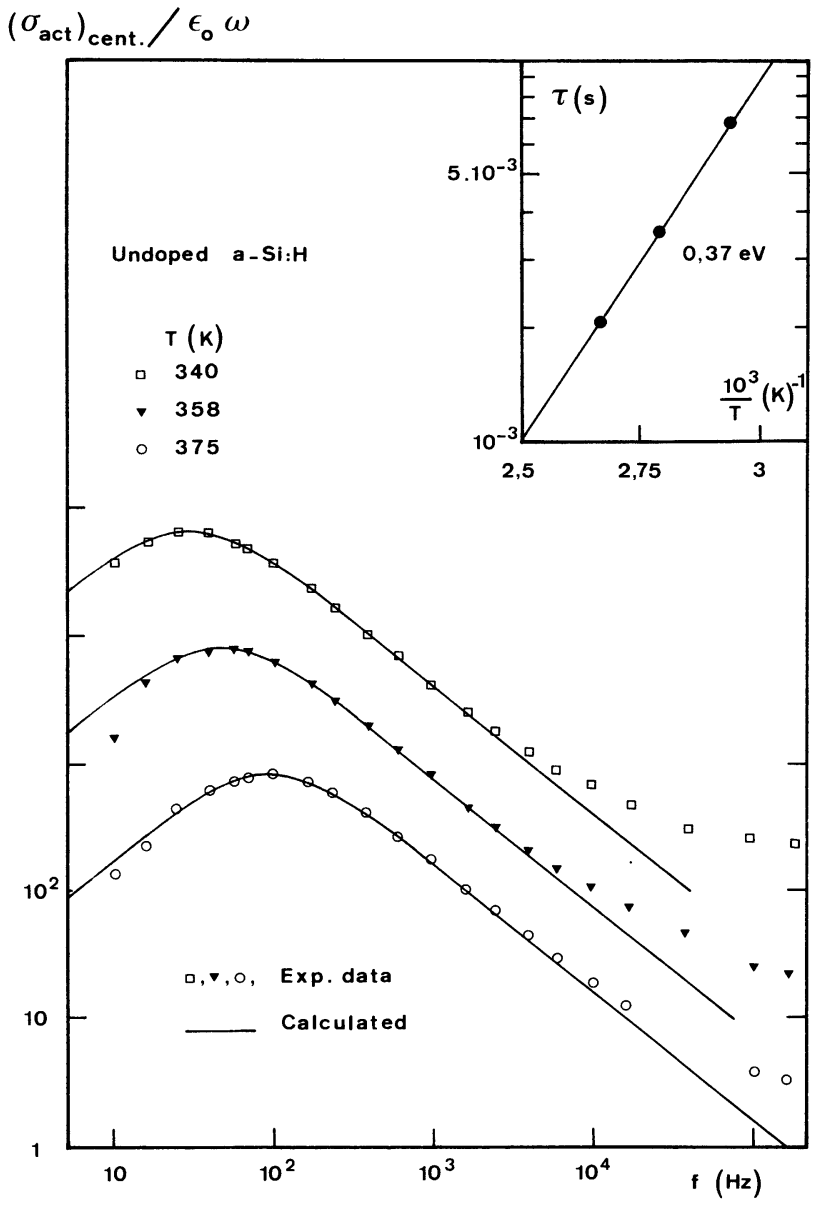

Fig. 6. - Experimental $\left(\sigma_{\mathrm{ac}}\right)_{\text {cent }} / \varepsilon_{0} \omega$ data versus frequency $(\square, \nabla \ldots)$ for undoped a-Si : H ; (一) calculated curves; the ordinate scale corresponds to the $375 \mathrm{~K}$ curve; the two other curves have been shifted along the ordinate axis (factors 10 and $10^{2}$ respectively). Insert : Arrhenius plot of $\tau_{4}(1 / T)$.

$$
\begin{aligned}
& N_{\mathrm{t} 1}=3.3 \times 10^{22} \mathrm{~m}^{-3} \\
& N_{\mathrm{t} 2}=1.6 \times 10^{23} \mathrm{~m}^{-3} \\
& N_{\mathrm{t} 3}=3.3 \times 10^{23} \mathrm{~m}^{-3} \\
& N_{\mathrm{t} 4}=7 \times 10^{23} \mathrm{~m}^{-3}
\end{aligned}
$$

The agreement between experimental and calculated values of both $\sigma_{\text {act }}(\omega, T)$ and $\varepsilon_{\text {act }}(\omega, T)$ is quite remarkable. Some levels (visible in figure 3 between 210 and $256 \mathrm{~K}$ around $100 \mathrm{~Hz}$, and above $340 \mathrm{~K}$ at $10^{4} \mathrm{~Hz}$ ) were neglected because their contribution, in the temperature range of experiment, is about two orders of magnitude lower than that of the main centers. The temperaure dependence of $\left(\varepsilon_{\mathrm{LF}}-\varepsilon_{\mathrm{HF}}\right)_{\text {cent }} / \varepsilon_{0}$ is compared to the calculated curve in figure 7 which also shows the individual contributions of the four centers.

It is observed (Figs. 3, 7) that $\varepsilon_{\mathrm{LF}}$ increases with $T$ at low temperatures, becomes maximum around $250 \mathrm{~K}$; then decreases, becomes minimum near 


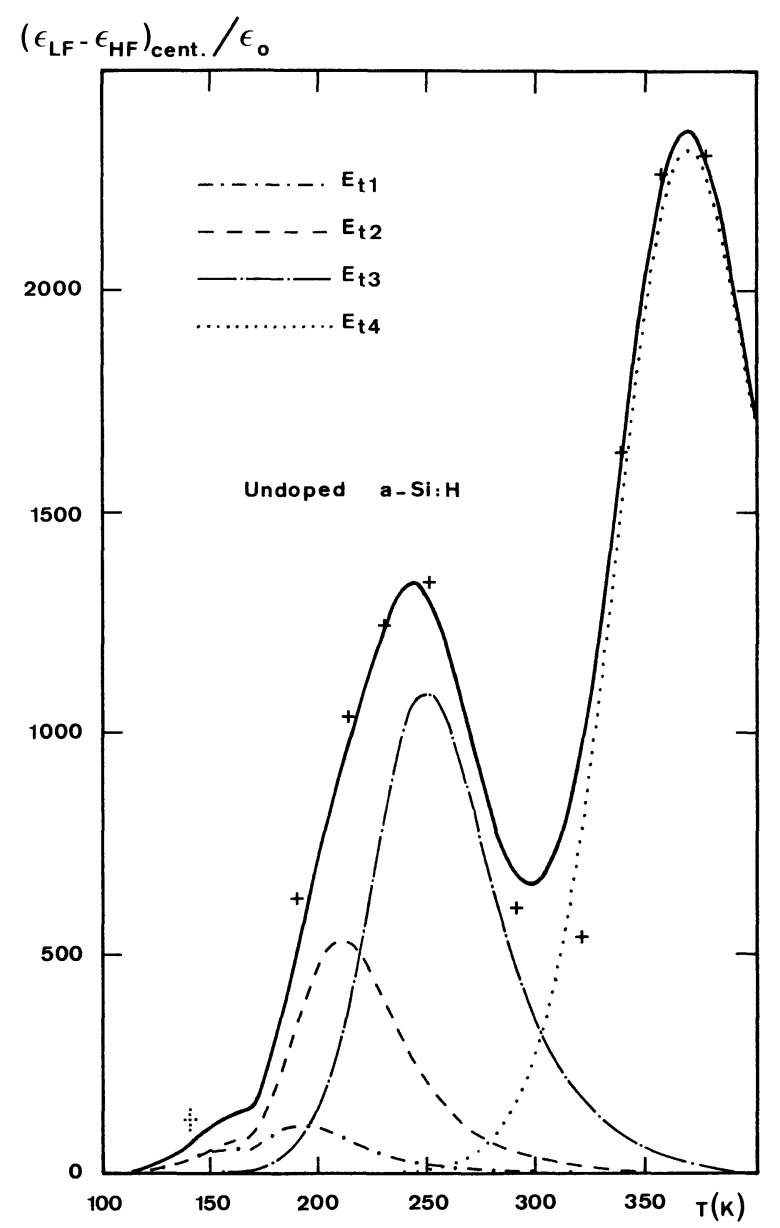

Fig. 7. - Full line : calculated values of $\left(\varepsilon_{\mathrm{LF}}-\varepsilon_{\mathrm{HF}}\right)_{\mathrm{cent}} / \varepsilon_{0}$ versus temperature (Eq. (31)) of undoped a-Si : $\mathrm{H}$. Dotted lines : contributions of individual centers.

$300 \mathrm{~K}$, and increases again. This behavior, quite unexplainable by theories ignoring the existence of $\mathrm{PF}$, is accurately reproduced in the calculated curves (Fig. 7). It results from the shift of the Fermi level relatively to the deep levels in the wells of maximum magnitude. According to section 2, the contribution of a deep center to the complex conductivity is significant only when its ionization probability changes with the external field. This happens when the energy of the level coincides, within $k T$, with the Fermi level at the bottom of deepest wells, because only the maximum magnitude $\Gamma g(\varepsilon)$ of PF depends on the external field. In order to demonstrate clearly what happens, figure 8 shows the location of the Fermi level relative to the center energy at five temperatures above $188 \mathrm{~K}$ (in this range $g_{0} \Gamma$ is independent of temperature). The contributions to $\left(\varepsilon_{\mathrm{LF}}-\varepsilon_{\mathrm{HF}}\right)_{\text {cent }}$ of centers $\mathrm{n}^{\circ} 1$ and 2 (Fig. 7) are maximum at $195 \mathrm{~K}$ and $205 \mathrm{~K}$ respectively, because at these temperatures the Fermi level coincides nearly with energy levels $n^{\circ} 1$ and 2 at the bottom of the wells (Fig. 8). Above $210 \mathrm{~K}$ the contributions of these centers decrease, and that of centers $n^{\circ} 3$ to $\left(\varepsilon_{\mathrm{LF}}-\varepsilon_{\mathrm{HF}}\right)_{\mathrm{cent}}$ becomes predominant and is maxi-

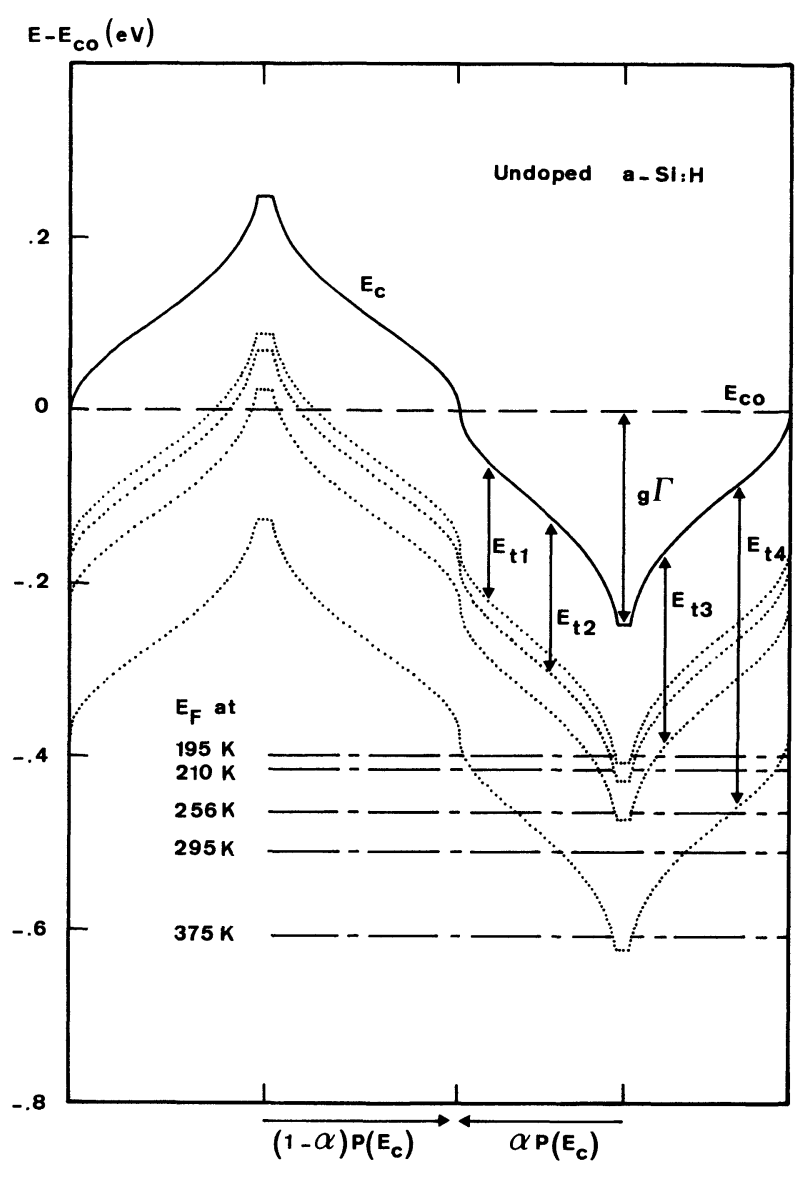

Fig. 8. - Band edge, deep centers, and Fermi level at five temperatures, versus probability $(1-\alpha) P\left(E_{\mathrm{c}}\right)$ or $\alpha P\left(E_{\mathrm{c}}\right)$, in undoped a-Si : $\mathrm{H}$.

mum near $256 \mathrm{~K}$ : at this temperature the energy of this center coincides with $E_{\mathrm{F}}$ at the bottom of the wells (Fig. 8), while centers $n^{\circ} 1$ and 2 which are at 3 and $2 k T$ above $E_{\mathrm{F}}$ respectively no longer play a noticeable role. The contribution of center $n^{\circ} 3$ decreases above $256 \mathrm{~K}$, and the contribution of center $\mathrm{n}^{\circ} 4$ starts to increase rapidly, as $E_{\mathrm{c} 0}-E_{\mathrm{F}}$ increases with $T$. So $\left(\varepsilon_{\mathrm{LF}}-\varepsilon_{\mathrm{HF}}\right)_{\text {cent }}$ goes through a minimum at $295 \mathrm{~K}$, increases again with $T$, and becomes maximum around $375 \mathrm{~K}$ where center $\mathrm{n}^{\circ} 4$ is close to $E_{\mathrm{F}}$ at the bottom of the wells. Therefore the variation of $\left(\varepsilon_{\mathrm{LF}}-\varepsilon_{\mathrm{HF}}\right)_{\text {cent }}$ versus temperature is quite accurately explained by the temperature dependence of $E_{c 0}-E_{\mathrm{F}}$ calculated from the fit of the band conductivity (Eqs. 7, 18). Moreover the calculation shows that, in the low temperature range, $E_{\mathrm{F}}$ is located $0.1 \mathrm{eV}$ below $E_{\mathrm{c}}$ at the bottom of the deepest wells at $110 \mathrm{~K}$, and at $0.12 \mathrm{eV}$ at $140 \mathrm{~K}$ (Fig. 9). So $E_{\mathrm{F}}$ is then intersecting the four levels largely above the bottom of the wells. The fact that, according to figures 3 and 7 , the contribution of the centers to $\left(\varepsilon_{\mathrm{LF}}\right)_{t}$ is very small in this temperature range confirms clearly that $\Gamma$ is independent of $\varepsilon$, and that only $g$ varies vith the applied field. Due to the very rapid variation of $\partial N_{t i}^{+} / \partial g$ when a level $i$ 


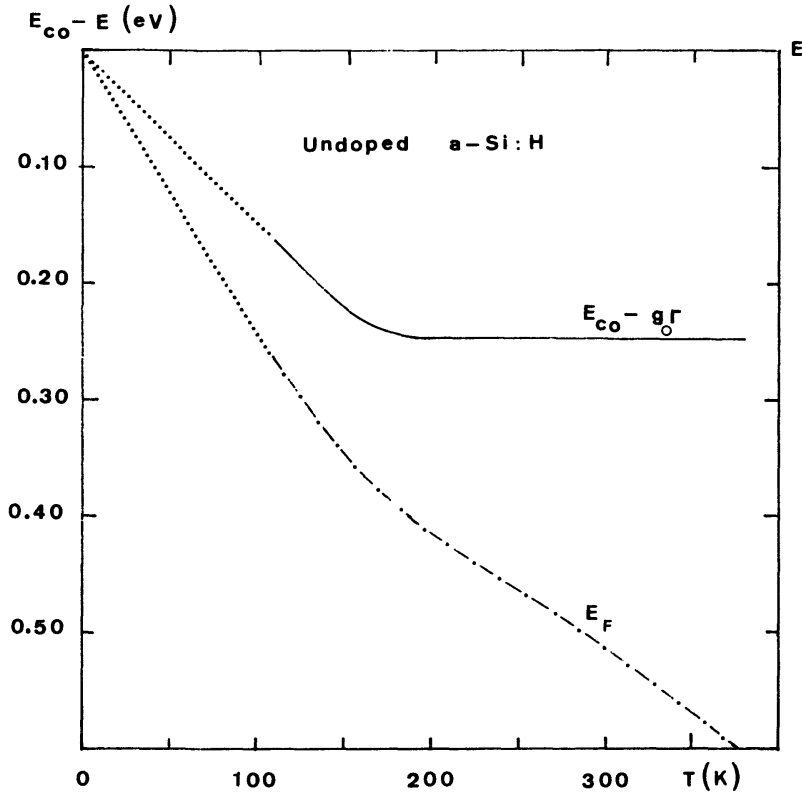

Fig. 9. - Energy $E_{c 0}-g_{0} \Gamma$ of the deepest wells, and Fermi level versus temperature in undoped a-Si : $\mathrm{H}$.

crosses $E_{\mathrm{F}}$ at the bottom of deepest wells, the uncertainty on $E_{\mathrm{t} i}$ is likely smaller than $k T$. A change of $5 \%$ in the energy of a peak generally prevents from fitting the data; for example the changes produced by reducing $E_{\mathrm{t} 3}$ from 0.225 to $0.215 \mathrm{eV}$ are shown in figure 10 . For the same reason, the possible broadening of the peaks in the density of states does not likely exceeds $5 \%$ of the peak energy.

It must be noticed that the temperature dependence of $g_{0}$ mentioned above is deduced from the fit of $\sigma_{\mathrm{dc}}$ data versus $10^{3} / T$ (Fig. 11) and from the fit of $\sigma_{\text {ac }}$ due to band carriers (Fig. 4). It is interesting to note that the two apparent $\sigma_{\mathrm{dc}}$ " activation energies » $E_{1} \simeq 0.12 \mathrm{eV}, \quad E_{2} \simeq 0.24 \mathrm{eV}$ which are observed (Fig. 11) correspond to the temperature ranges in which $g_{0}$ is proportional to $T$, and independent of $T$ respectively, but not to any of the actual activation energies of the centers. This fact, previously mentioned $[15,17]$, shows how a crude interpretation of Arrhenius dc conductivity diagrams can lead to quite erroneous conclusions when ignoring the existence of PF.

The average electron density in the band $\sigma_{\infty} / q \mu_{\mathrm{n}} \simeq 7.2 \times 10^{18} \mathrm{~m}^{-3}$ is very small compared to the total density $\sum_{i} N_{\mathrm{t} i} \simeq 1.2 \times 10^{24} \mathrm{~m}^{-3}$ of the four (almost completely ionized) levels observed between $110 \mathrm{~K}$ and $375 \mathrm{~K}$. Therefore the material remains nearly exactly compensated in this range : $\sum_{i} N_{\mathrm{d} i}^{+} \simeq$ $\sum_{j} N_{\mathrm{aj}}^{-}$. This is possible, as shown by numerical calculations, if donor and acceptor levels are intercalated, more or less regularly. The contribution of a

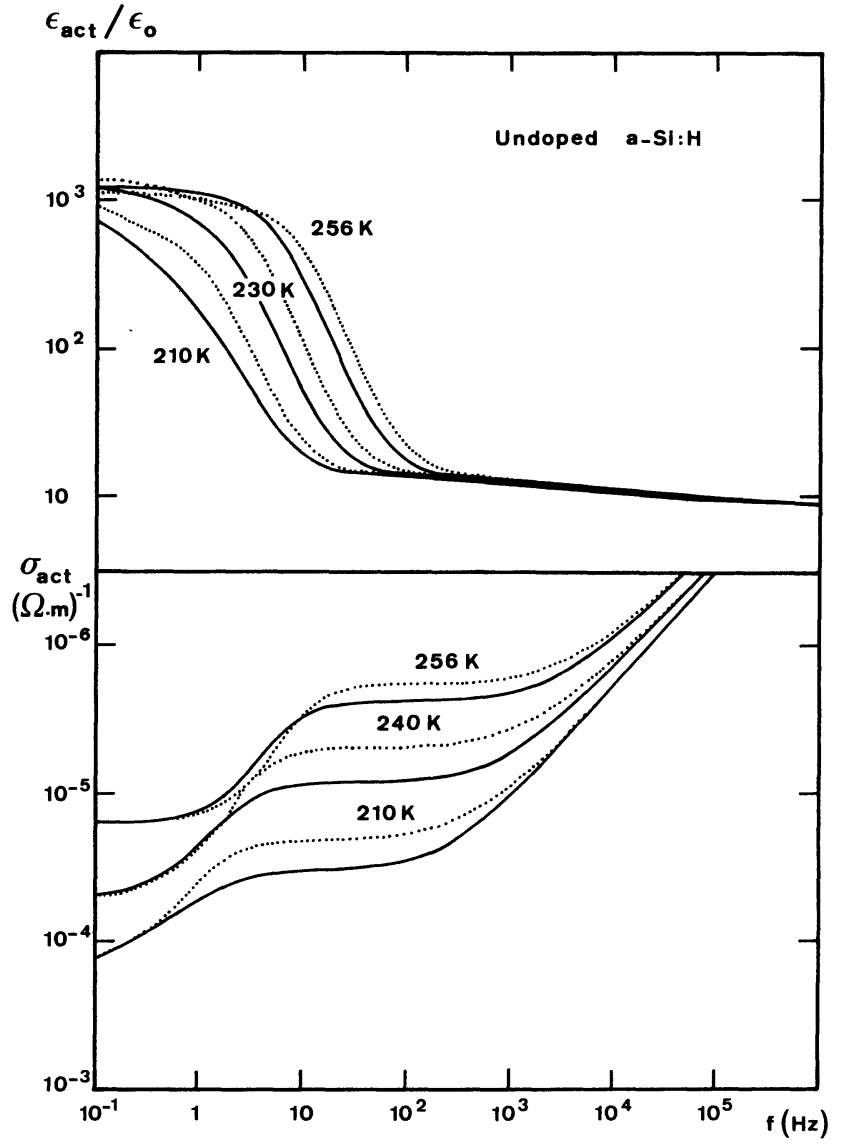

Fig. 10. - Comparison of calculated $\sigma_{\text {act }}$ and $\varepsilon_{\text {act }}$ isotherms with $E_{\mathrm{d} 3}=0.225 \mathrm{eV}$ (full line), and $E_{\mathrm{d} 3}=0.215 \mathrm{eV}$ (dotted line).

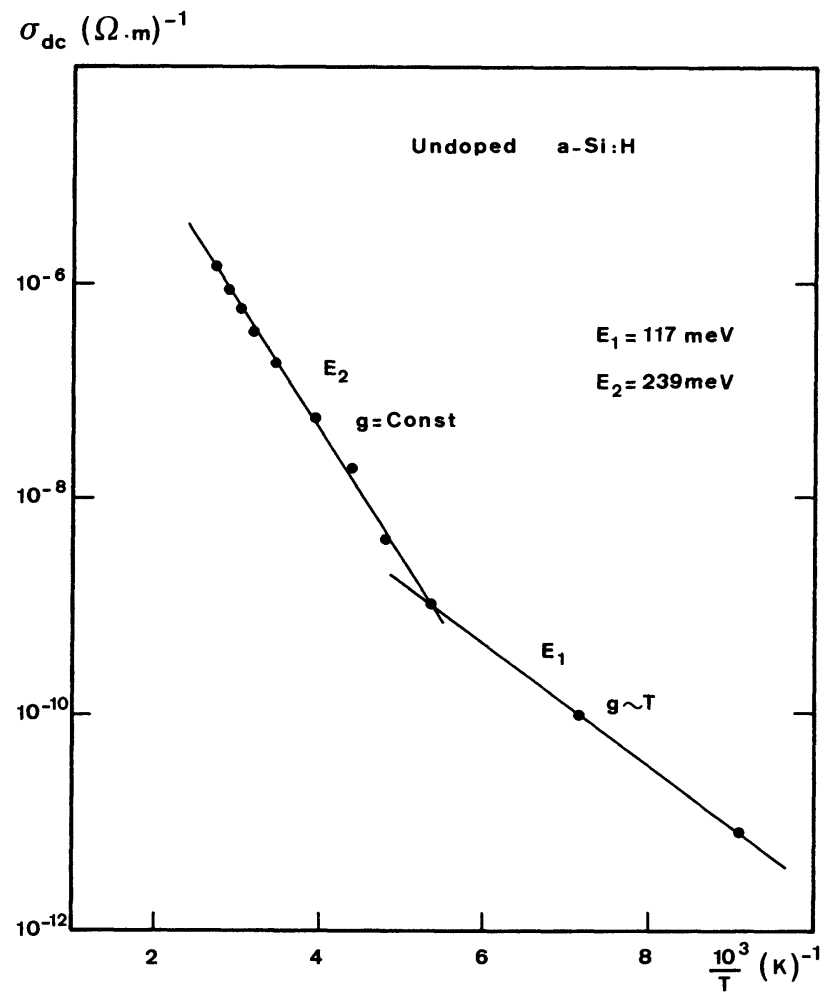

Fig. 11. - Experimental $\sigma_{\mathrm{dc}}$ data versus $1 / T$ for undoped a-Si : $\mathrm{H}$. 
level to $\Delta N_{\mathrm{d} i}^{+}$(or $\Delta N_{\mathrm{a} j}^{-}$) resulting from the shift of $E_{\mathrm{F}}(T)$ is maximum when $E_{\mathrm{c} 0}-E_{\mathrm{F}} \simeq E_{\mathrm{d} i}\left(\right.$ or $\left.E_{\mathrm{aj}}\right)$, while its contribution to $\sigma_{\text {ac cent }}$ and $\varepsilon_{\text {ac cent }}$ is maximum when $E_{\mathrm{c} 0}-E_{\mathrm{F}}=g \Gamma+E_{\mathrm{d} i}\left(\right.$ or $E_{\mathrm{aj}}$ ). So, it is clear (Fig. 8) that, above $256 \mathrm{~K}$, deeper levels (which do not yet affect significantly $\sigma_{\text {ac cent }}$ and $\varepsilon_{\text {ac cent }}$ as mentioned previously) begin to contribute to the compensation of level $n^{\circ} 4$.

ii) Boron doped $\mathrm{p}$ type sample : this $\mathrm{p}$ type sample has a much larger conductivity than the undoped one, so the measurements could be performed from $8 \mathrm{~K}$ to $298 \mathrm{~K}$, allowing the gap state density to be determined near the valence band edge. The experimental $\sigma_{\text {act }}$ and $\varepsilon_{\text {act }} / \varepsilon_{0}$ data $v$ s. $\omega$ are plotted in figures 12,13 ; the full lines correspond to the calculated values. The existence of a series of peaks in the density of states is proved by the existence of a set of maxima in the $\sigma_{\text {act }} / \varepsilon_{\text {act }} \omega$ isotherms. The fitting procedure is the same as before. However $\sigma_{\infty}(T)$ is approximately known by the saturation of the $\sigma_{\text {act }}(\omega)$ curves above $10^{5} \mathrm{~Hz}$. Further adjustments of $\sigma_{\infty}(T)$ are made in order to obtain the best fit. In this sample, $\Gamma$ is equal to $45.8 \mathrm{meV}$, only one third of the value found in the undoped sample, and $\alpha=0.934$. As $T$ increases $g_{0} \Gamma$ increases linearly up to $24.5 \mathrm{~K}$ (Fig. 14), then the slope decreases between 24.5 and $150 \mathrm{~K}$; above $200 \mathrm{~K}, g$ remains constant and equal to $g_{0}=2.17$. The variation of $E_{\mathrm{F}}-E_{\mathrm{v} 0}$ versus $T$ ensued from the fit is also shown in

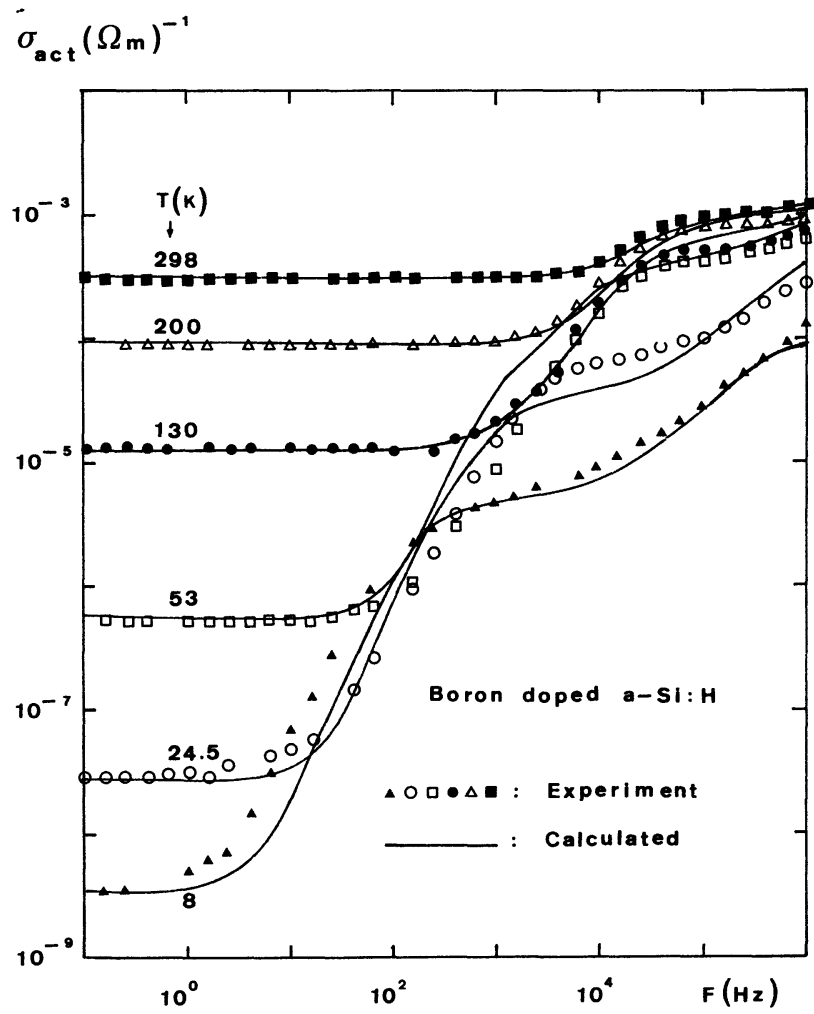

Fig. 12. - Conductivity $\sigma_{\text {act }}(T)$ versus frequency of Boron doped a-Si : H : $(\boldsymbol{\square}, \triangle \ldots)$ Experiment ; $(\longrightarrow)$ Calculated values (Eqs. $(12,13,28)$ ).

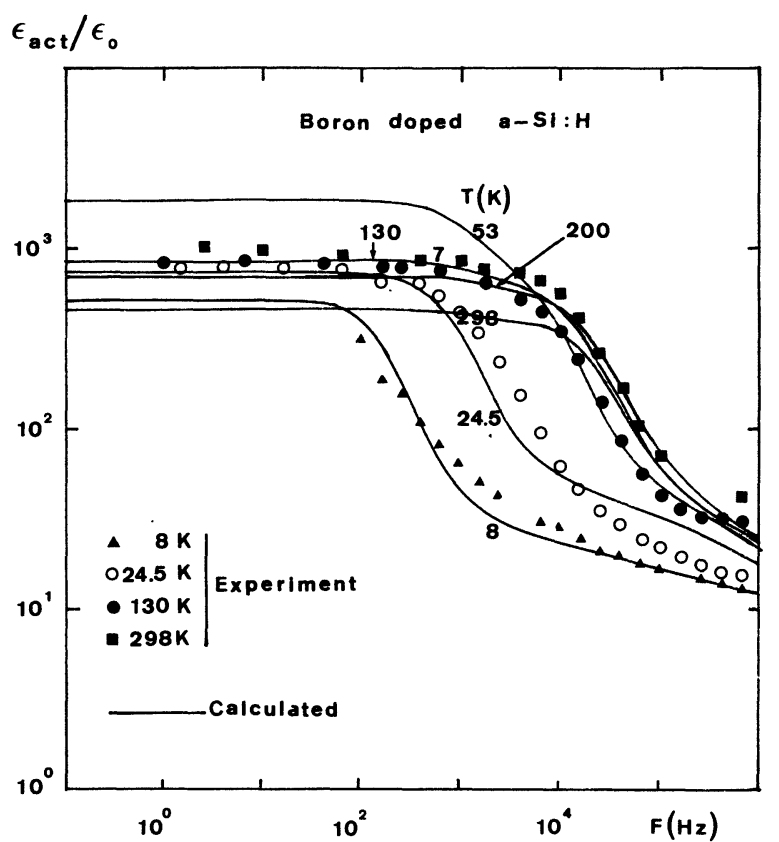

Fig. 13. - Relative permittivity $\varepsilon_{\text {act }}(T) / \varepsilon_{0}$ versus frequency of boron doped a-Si : $\mathrm{H}:(\boldsymbol{\square}, \bullet, \mathrm{O} \ldots)$ Experiment ; (—) Calculated values (Eqs. $(12,14,29))$.

figure 14. As is expected [17], in the low temperature range where $g_{0} \Gamma$ increases linearly with $T$, the Fermi level stays close to the bottom of the deepest wells : $E_{\mathrm{F}}-E_{\mathrm{v} 0} \simeq g_{0} \Gamma$. In fact, the calculated values of $E_{\mathrm{F}}-E_{\mathrm{v} 0}$ are smaller than $g_{0} \Gamma$ by an amount which does not exceed $k T$, so that the resulting error in using non-degenerate statistics for calculating the carrier density at the bottom of the deepest wells is very small. It is found that $\sigma_{\infty}$ increases from

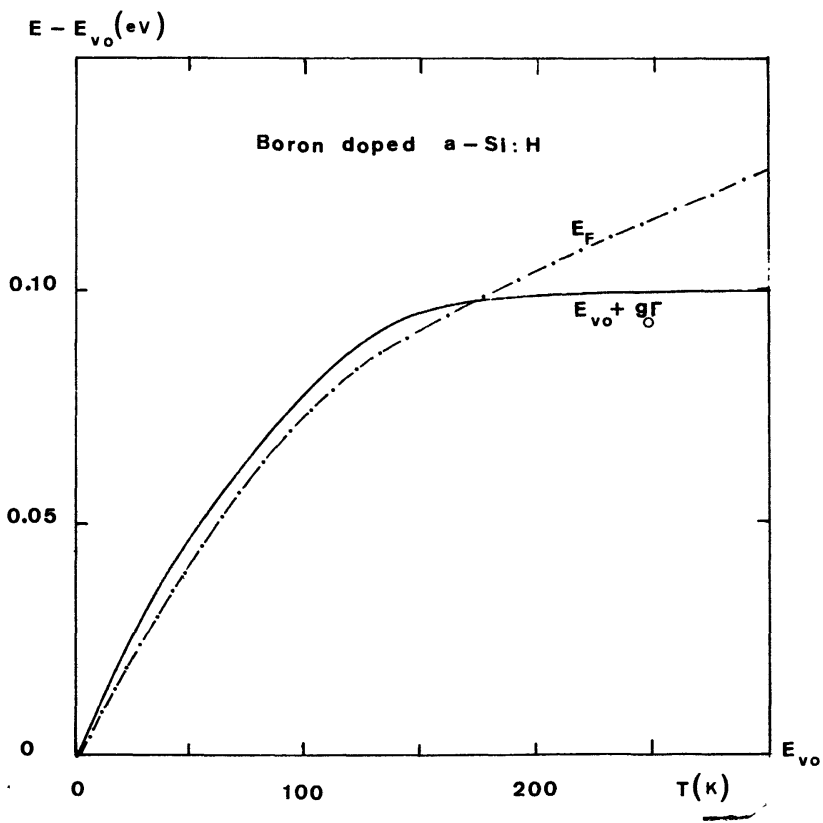

Fig. 14. - Energy $E_{\mathrm{v} 0}+g_{0} \Gamma$ of the highest hills, and Fermi level, versus temperature in boron doped a-Si : $\mathrm{H}$. 
$1 \times 10^{-4}(\Omega \mathrm{m})^{-1}$ at $8 \mathrm{~K}$ to $7.25 \times 10^{-4}(\Omega \mathrm{m})^{-1}$ at $298 \mathrm{~K}$, and that the hole mobility $\mu_{\mathrm{p}}$ varies from $2.5 \times 10^{-9}$ to $6 \times 10^{-9} \mathrm{~m}^{2} / \mathrm{Vs}$. This mobility is about $10^{4}$ to $10^{5}$ times smaller than the mobility of electrons in the undoped sample. The hole density $p=\sigma_{\infty} / q \mu_{\mathrm{p}}$ increases from $2.5 \times 10^{23} \mathrm{~m}^{-3}$ at $8 \mathrm{~K}$ to $6 \times 10^{23} \mathrm{~m}^{-3}$ at room temperature. This carrier density is about $10^{5}$ times larger than in the undoped material. The experimental $\sigma_{\mathrm{dc}}(\boldsymbol{\varepsilon})$ data are plotted in figure 15. At each temperature we try to find

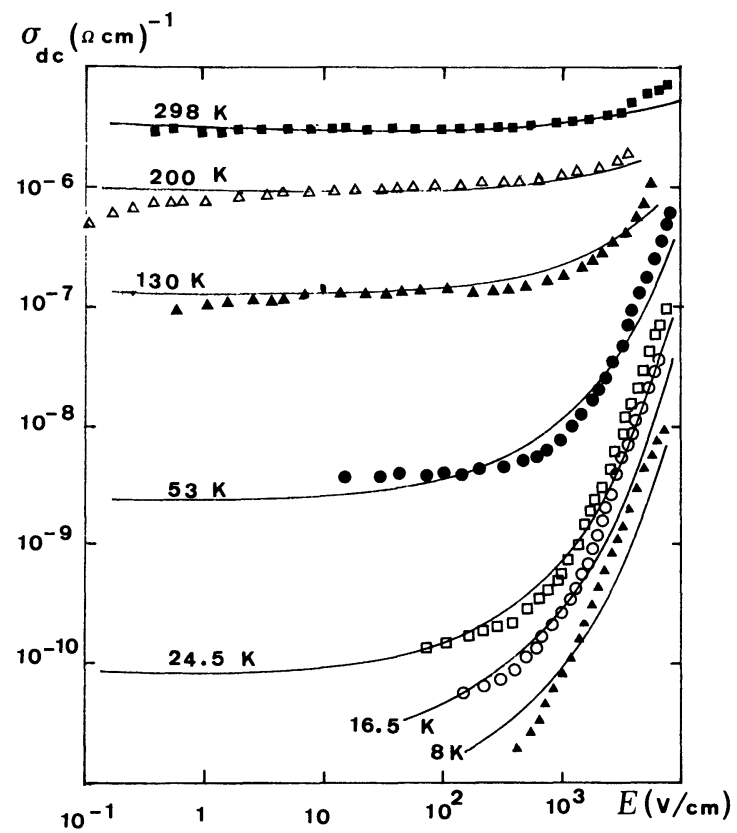

Fig. 15. - dc conductivity $\sigma_{\mathrm{dc}}$ versus electric field in boron doped a-Si : H : $(\boldsymbol{\square}, \Delta, \Delta \ldots)$ Experiment ; ( $\longrightarrow)$ Calculated values (Eqs. $(19,20))$.

$g(\mathcal{E})$ corresponding to the data (Eq. 19), and we plot $\frac{g_{0}-g}{g_{0}}$ versus $\varepsilon^{1 / 2}$. An example is given for $T=70 \mathrm{~K}$ in figure 16, which shows that the points are reasonable close to a straight line. According to equation (20) the slope of this line is equal to $\varepsilon_{\mathrm{c}}^{-1 / 2}$. The $\varepsilon_{\mathrm{c}}(T)$ values range between 1.5 and $3 \times 10^{6} \mathrm{~V} / \mathrm{m}$, and there is no clear evidence of an activation energy. Then we use a constant value $\varepsilon_{\mathrm{c}}=2 \times 10^{6} \mathrm{~V} / \mathrm{m}$ in order to calculate $\partial g / \partial \mathcal{E}$, with a possible error less than $30 \%$. In figure 15 the calculated $\sigma_{\mathrm{dc}}(\varepsilon)$ curves are compared to the experimental data. Between 8 and $53 \mathrm{~K}$ the values of $g_{0}$ leading to the best fit are $10 \%$ larger than those obtained by fitting the $\sigma_{\text {act }}(\omega, T)$ data. This small discrepancy is likely due to the difficulty, in successive low temperature experiments, to reproduce exact equilibrium conditions with the same charges on the centers.

The evidence of a series of peaks in the gap state density is demonstrated by plotting experimental

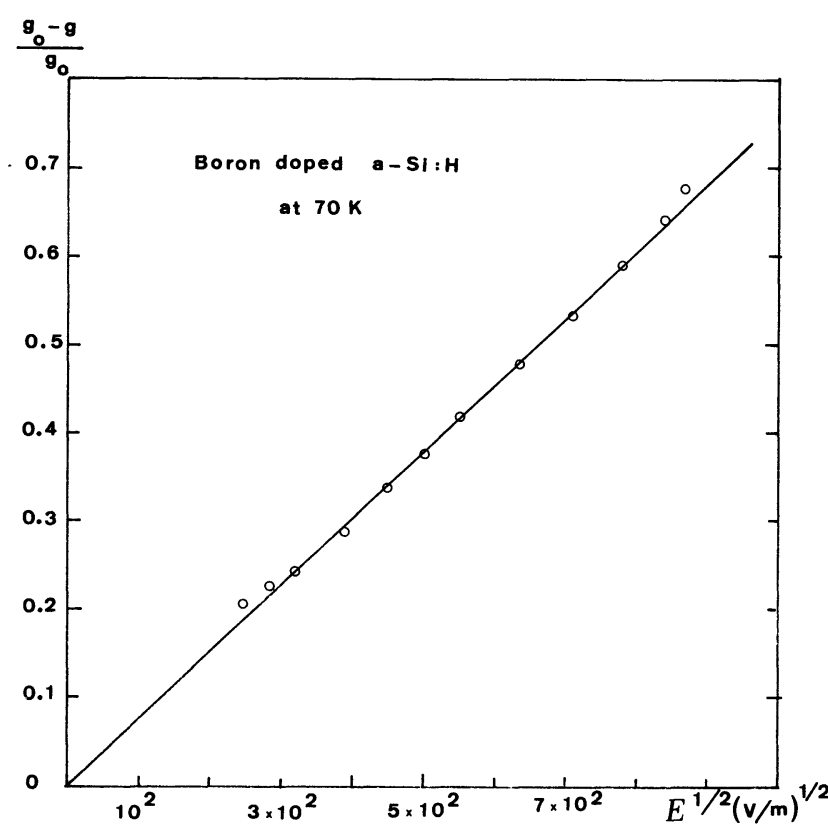

Fig. 16. $-\left(g_{0}-g\right) / g_{0}$ versus $\varepsilon^{1 / 2}$ in boron doped a-Si : H ; (O) Experiment.

$\sigma_{\mathrm{act}} / \varepsilon_{0} \omega$ isotherms. A first level of low binding energy is observed in the very low temperature range, but the lack of experimental data below $8 \mathrm{~K}$ prevents a precise determination of $\tau_{10}$ and $E_{\mathrm{a} 1}$. A second center becomes predominant between 24.5 and $53 \mathrm{~K}$ (Fig. 17); from the Arrhenius plot of $\tau_{2}$ versus $T^{-1}$ (insert of Fig. 17) we find: $\tau_{20} \simeq$ $4 \times 10^{-6} \mathrm{~s} ; E_{\mathrm{a} 2} \simeq 7 \mathrm{meV}$. Above $130 \mathrm{~K}$ the contribution to the dielectric losses of a third center becomes dominant. The Arrhenius plot of $\tau_{3}$ versus $T^{-1}$ (insert Fig. 17) leads to: $\tau_{30} \simeq 5 \times 10^{-6} \mathrm{~s}$; $E_{\mathrm{a} 3} \simeq 13 \mathrm{meV}$. The characteristics of these three peaks are adjusted by successive approximations, as before, until the best fit of experimental data is obtained. The full lines in figures 12 and 13 are calculated by using the following physical characteristics of the centers (the subscript a is used even if it is not sure that all these centers are acceptors) :

$$
\begin{gathered}
E_{\mathrm{a} 1}=1.46 \mathrm{meV}, \quad \tau_{10}=1.04 \times 10^{-4} \mathrm{~s}, \\
E_{\mathrm{a} 2}=6.83 \mathrm{meV}, \quad \tau_{20}=4.0 \times 10^{-6} \mathrm{~s}, \\
E_{\mathrm{a} 3}=12.89 \mathrm{meV}, \quad \tau_{30}=5.1 \times 10^{-6} \mathrm{~s}, \\
N_{\mathrm{a} 1}=5 \times 10^{23} \mathrm{~m}^{-3} ; \\
N_{\mathrm{a} 2}=1 \times 10^{24} \mathrm{~m}^{-3} ; \\
N_{\mathrm{a} 3}=1 \times 10^{24} \mathrm{~m}^{-3} .
\end{gathered}
$$

The hole density $\mathrm{p}=\sigma_{\infty} / q \mu_{\mathrm{p}}$ deduced from the value of $\sigma_{\infty}$, and the acceptor level densities fitting the contribution of these centers to $\left(\sigma_{\mathrm{ac}}\right)_{\text {cent }}$ and $\left(\varepsilon_{\mathrm{ac}}\right)_{\text {cent }}$, are determined independently. The fact that $\mu_{\mathrm{p}}$ has the right value can be proved with the aid of the average neutrality equation (6). At $8 \mathrm{~K}$, 
$g_{0} \Gamma=8.26 \mathrm{meV}$ is much smaller than $\Gamma$, so PF's of maximum magnitude occupy most of the sample. At this temperature the first level is partly ionized, the other two being nearly neutral. The hole density $p=2.5 \times 10^{23} \mathrm{~m}^{-3}$ is consistent with the density $N_{\mathrm{a} 1}=5 \times 10^{23} \mathrm{~m}^{-3}$. At room temperature $E_{\mathrm{F}}-E_{\mathrm{v} 0}=125.7 \mathrm{meV}$ while $g_{0} \Gamma=99.4 \mathrm{meV}$ so the first and, to a lower degree, the second levels are nearly fully ionized; this is in rough agreement with the calculated hole density equal to $6 \times 10^{23} \mathrm{~m}^{-3}$.

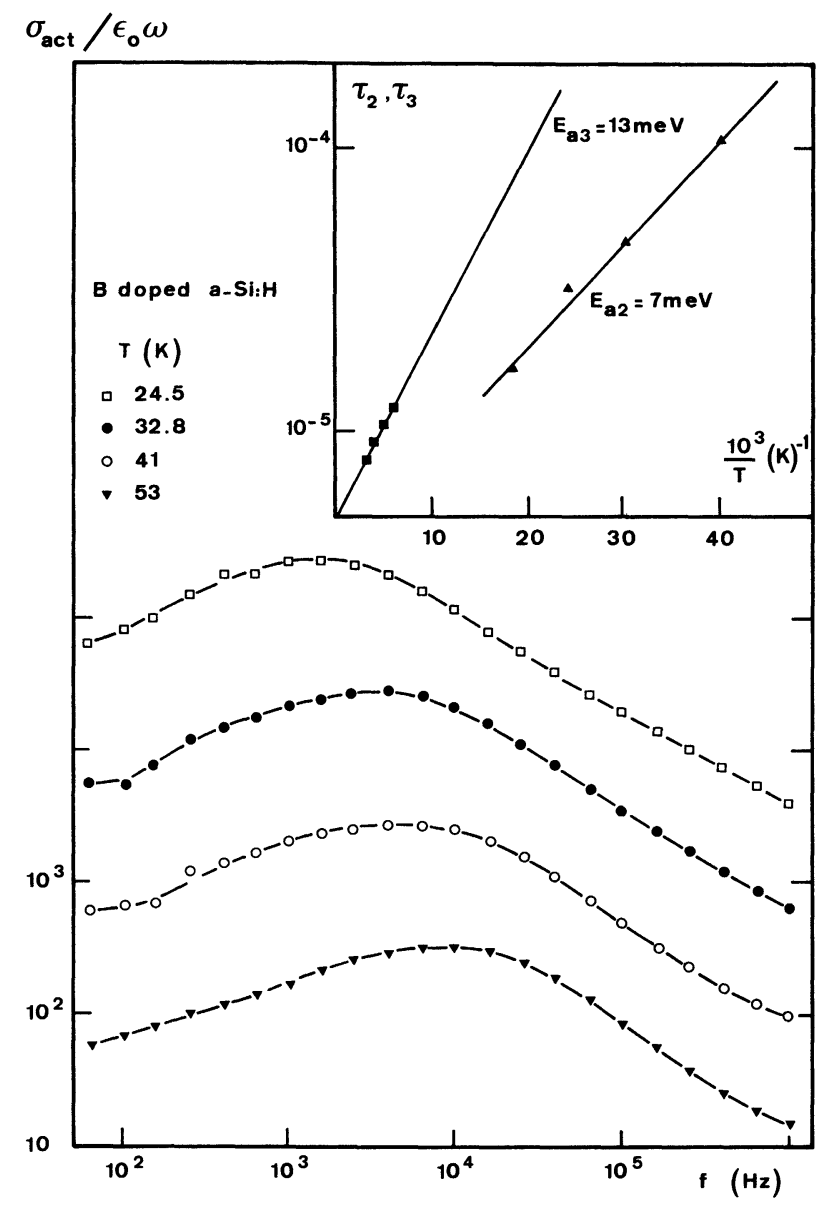

Fig. 17. - Experimental $\sigma_{\text {act }} / \varepsilon_{0} \omega$ versus $\omega$, near $\omega_{2}=1 / \tau_{2}$ for boron doped a-Si : $\mathrm{H}$. The ordinate scale corresponds to the $53 \mathrm{~K}$ curve. The other curves have been shifted along the ordinate axis (factors 10,102,103). Insert : Arrhenius plot of $\tau_{2}, \tau_{3}$.

Therefore the value of the mobility is correct. On the other hand the possible continuous density of gap states between the peaks is likely much lower than the peak density, because otherwise the data could not be correctly fitted.

3.3 Discussion. - Let us discuss the foregoing results :

i) The analysis of experimental data reveals that undoped a-Si : $\mathrm{H}$ is a highly compensated material in which PF's of large magnitude are found. The boron doped a-Si : $\mathrm{H}$ sample is much less compensated and consequently $\Gamma$ is significantly smaller. Systematic investigation would be needed in order to know whether or not $\Gamma$ is characteristic of the material and of the doping level, or if it also depends on the growth conditions. This could help to improve the growth and doping techniques.

ii) The gap state density, in our undoped and doped samples, is constituted by a discrete series of well resolved peaks, contrary to the usual model assuming a continuous ditribution of band tail states. Such a continuous distribution of states is possibly superposed to the observed peaks, but it is not distinguishable in our experiments, and is therefore certainly of low density compared to that of the peaks. It must be noticed that the methods generally used in the literature do not provide precise information on the gap state density, because they do not take into account the existence of PF, and they often are unable to distinguish, as is shown above for F.E., the separate contributions of band carriers and of shallow states. So, when PF are ignored, F.E. methods lead to overevaluated values of the density of states, particularly when approaching the band edges ; similarly the validity of the use of S.C.L.C. theory without taking into account the effect of image force barrier lowering on dc band conductivity is certainly questionable. On the other hand, in the case of the sweep-out technique $[6,7]$, the measured current likely results from the erasing of PF by the applied field [29]. It corresponds [40] to the return to quasi equilibrium of some fraction of the shallow centers, which depends on the compensation and is therefore difficult to evaluate.

iii) In the doped sample, the first discrete peaks are located at a few meV from the band edge of the extended states occupied by free holes, so that there is no evidence of a mobility edge distinct from the energy band edge. The concept of a mobility gap was introduced in order to interpret dc data when ignoring the existence of PF, but our results show that this concept is probably not essential when the effects of PF are taken into account.

iv) In the doped sample, the average carrier density and the density of ionized shallow centers are similar. Center $n^{\circ} 1$ is probably an acceptor due to boron impurity, but in the absence of other information, nothing can be said on the type of the other centers.

v) Within the limits of error of the experiments and of the fit of the data, there is no evidence of hopping conduction; hopping conductivity is certainly at least a few orders of magnitude lower than band carrier conductivity, even in the undoped samples. 


\section{Conclusion.}

In the present paper, it is shown that PF of maximum magnitude play a significant role, particularly in the degree of ionization of deep centers. Generalizing the PRA theory, and taking into account the lowering of PF of maximum magnitude by the external field, general expressions of a.c. conductivity and permittivity of a bulk material, and of differential surface capacitance of a MIS diode are derived. The theory leads to a new method for evaluating the band carrier density and the gap state density, in amorphous and compensated semiconductors. Original conductivity and permittivity data on undoped and boron doped a-Si:H performed in unusually large ranges of temperatures and frequencies are reported. The remarkable agreement observed with the theory could not be reached when using existing models ignoring the existence of PF. This constitutes a strong argument in favour of the method developed in this paper. It is found that the density of shallow states or moderately deep states consists of a series of discrete peaks. The magnitude of PF is larger in highly compensated undoped samples than in boron doped ones. It is suggested that this method could be used to study the possible influence of growing and doping processes on the magnitude of PF and the density of states.

\section{Acknowledgment.}

We express our gratitude to D. Kaplan, J. Magariño, A. Friederich, at Thomson-CSF, who kindly supplied the samples, and to S. Bennegadi for his contribution to this work.

\section{References}

[1] Debye, P., Ber. D. Phys. Ges. 15 (1913) 777.

[2] Cole, K. S., Cole, R. H., J. Chem. Phys. 9 (1941) 341.

[3] Davidson, D. W., Cole, R. H., J. Chem. Phys. 19 (1951) 1484.

[4] Jonscher, A. K., Phys. Thin Films 11 (1980) 202.

[5] Macdonald, J. R., J. Appl. Phys. 61 (1987) 700.

[6] Street, R. A., Philos. Mag. B 50 (1984) L19; Phys. Rev. B 32 (1985) 3910.

[7] Street, R. A., Kakalios, J., Tsai, C. C., Hayes, T. M., Phys. Rev. B 35 (1987-II) 1316.

[8] Fritzsche, H., J. Non-Cryst. Sol. 77-78 (1985) 273.

[9] Wannier, G. H. Phys. Rev. 76 (1949) 438.

[10] Shockley, W., Bardeen, J., Phys. Rev. 77 (1950) 407.

[11] Fritzsche, H., J. Non-Cryst. Solids 6 (1971) 49.

[12] Shklovskil, B. I., Efros, A. L., Sov. Phys. JETP 33 (1971) 468.

[13] ShklovskiI, B. I., Efros A. L., Sov. Phys. JETP 35 (1972) 610.

[14] Pistoulet, B., Robert, J. L., Dusseau J. M., EnsuQue, L., J. Non-Cryst. Sol. 29 (1978) 29.

[15] Pistoulet, B., Girard, P., Hamamdian, G., $J$. Appl. Phys. 56, a) (1984) 2268 ; b) (1984) 2275.

[16] Pistoulet, B., Hamamdian, G., Phys. Rev. B 35 (1987) 6305 ;

HAMAmDJiAN, G., Thesis Univ. Montpellier II, Jan. 1987.

[17] Pistoulet, B., Roche, F. M., Abdalla, S., Phys. Rev. B 30 (1984) 5987.

Roche, F. M., Thesis Univ. Montpellier II, Nov. 1984.

[18] Soegandi, T., Roche, F. M.,'Pistoulet, B., Solid State Commun. 55 (1985) 967.

[19] Pollak, M., Geballe, T. H., Phys. Rev. 122 (1961) 1742.

[20] Abkowitz, M., Le Comber, P. G., Spear, W. E., Commun. Phys. 1 (1976) 175.

[21] Long, A. R., Balkan, N., J. Non-Cryst. Solids 35$36(1980) 415$.

[22] Moustakas, T. D., Weiser, K., Phys. Rev. B 12 (1975) 2448.
[23] Pfister, G., Phys. Rev. Lett. 36 (1976) 271.

[24] Pistoulet, B., Girard, P., Roche, F. M., Physics of Disordered Materials, Eds. D. AdLER, H. FRITZSCHE, S. R. OvSHINSKY (Plenum Press N. Y. (1985) p. 425.

[25] Anderson, P. W., Phys. Rev. 109 (1958) 1492.

[26] MotT, N. F., DAvis, E. A., Electronic Processes in Non-Crystalline Materials (Oxford University Press) 2nd Edition (1979).

[27] Cohen, M. H., Fritzsche, H., Ovshinsky, S. R., Phys. Rev. Lett. 22 (1969) 1065.

[28] Keldysh, L. V., Proshko, G. P., Fiz. Tverd. Tela (Leningrad) 5 (1963) 12 ; Sov. Phys. Solid State 5 (1964) 12.

[29] Pistoulet, B., Abdalla, S., J. Appl. Phys. 60 (1986) 1059.

[30] Spear, W. E., Le Comber, P. G., J. Non-Cryst. Solids 8-10 (1972) 727.

[31] Madan, A., Le Comber, P. G., Spear, W. E., J. Non-Cryst. Solids 20 (1976) 239.

[32] Rehm, W., Kunzel, H., Dohler, G. H., Ploog, K., Ruden, P., Proceed. of the 16th Int. Conf. on the Physics of Semicond. Montpellier 1982, Ed. by M. Averous (North-Holland) Amsterdam (1982) p. 732.

[33] Abdalla, S., Pistoulet, B., J. Appl. Phys. 58 (1985) 2646.

[34] Pistoulet, B., Roche, F. M., Cagna, A., J. Phys. Colloq. France 42 (1981) C4-147.

[35] Jonscher, A. K., Electronic and Structural Properties of Amorphous Semiconductors, Eds. P. LE COMBER and J. MORT (Acad. Press, NY) (1973) p. 329.

[36] Zohta, Y., Solid State Electron. 16 (1973) 1029.

[37] Garrett, C. G. B., Brattain, W. H., Phys. Rev. 99 (1955) 376.

[38] Sol, N., Kaplan, D., Dieumegard, D., Dubreuil, D., J. Non-Cryst. Solids 35-36 (1980) 291.

[39] Magariño, J., Kaplan, D., Friederich, A., DeNeUville, A., Phil. Mag. 45 (1982) 285.

[40] Pistoulet, B., Abdalla, S., J. Appl. Phys. 63 (1988) 421. 